\title{
An In-Depth Exploration of Hospital-Based Physical and Occupational Therapists' Needs, Perceptions and Participation in a Safe Patient Handling and Mobility Program
}

\author{
Kimeran W. Evans \\ West Virginia University, kwevans@hsc.wvu.edu
}

Follow this and additional works at: https://researchrepository.wvu.edu/etd

Part of the Occupational Health and Industrial Hygiene Commons

\footnotetext{
Recommended Citation

Evans, Kimeran W., "An In-Depth Exploration of Hospital-Based Physical and Occupational Therapists' Needs, Perceptions and Participation in a Safe Patient Handling and Mobility Program" (2021). Graduate Theses, Dissertations, and Problem Reports. 8127.

https://researchrepository.wvu.edu/etd/8127

This Dissertation is protected by copyright and/or related rights. It has been brought to you by the The Research Repository @ WVU with permission from the rights-holder(s). You are free to use this Dissertation in any way that is permitted by the copyright and related rights legislation that applies to your use. For other uses you must obtain permission from the rights-holder(s) directly, unless additional rights are indicated by a Creative Commons license in the record and/ or on the work itself. This Dissertation has been accepted for inclusion in WVU Graduate Theses, Dissertations, and Problem Reports collection by an authorized administrator of The Research Repository @ WVU. For more information, please contact researchrepository@mail.wvu.edu.
} 
An In-Depth Exploration of Hospital-Based Physical and Occupational Therapists' Needs, Perceptions and Participation in a Safe Patient Handling and Mobility Program

\title{
Kimeran Evans
}

Dissertation submitted to the School of Public Health at West Virginia University

In partial fulfillment of the requirements for the degree of

$\mathrm{PhD}$ in Public Health

\author{
Douglas Myers, ScD, Chair \\ Weimin Gao, MS, MPH, PhD, Co-Chair \\ Kimberly Rauscher, ScD \\ Anna Allen, MD, MPH \\ Kathleen Rockefeller, PT, ScD, MPH
}

Occupational and Environmental Health Sciences

\author{
Morgantown, West Virginia \\ 2021
}

Keywords: occupational health, rehabilitation professionals, work related injuries, safe patient handling and mobility, healthcare workers, work related musculoskeletal disorders, hospital lift equipment 


\begin{abstract}
An In-Depth Exploration of Hospital-Based Physical and Occupational Therapists' Needs, Perceptions and Participation in a Safe Patient Handling and Mobility Program

Kimeran Evans

Introduction - Patient mobility performed in the hospital setting places healthcare workers at high risk for WMSDs. The healthcare industry has responded through the creation of SPHM programs that are successful in reducing work-related injuries broadly. However, less is known about the distinct experiences of rehabilitation professionals. Therefore, an in depth exploration of the perceptions, participation, and needs pertaining to lift equipment usage among rehabilitation professionals was performed.
\end{abstract}

Methods - A qualitative case study approach explored this bounded system. Six focus groups were conducted with 25 members of the rehabilitation team and three interviews were conducted with key administrative personnel. In addition, administrative documents related to the SPHM program were reviewed. Focus groups and interviews were recorded and de-identified transcripts and program documents were analyzed for emergent themes.

Results - Rehabilitation professionals were broadly supportive of SPHM programs. Participants provided suggestions for improvements to lift equipment to best fit within their patient mobility goals. They also denied work-related injuries but described experiencing work-related pain that they chose to self-manage. A dedicated SPHM training department and budgeting program and the performance of lift equipment audits were described as key administrative components.

Conclusion - Manufacturers should consider the equipment modifications suggested to maximize work safety. When measuring these program effects, injury records should not be used but rather information gained directly from the workers. SPHM administration should include an equipment audit process as well as a training program and budgeting program housed outside of each unit for program success and longev 


\section{Table of Contents}

\begin{tabular}{lll}
\hline Section & Content & Pages \\
\hline I & Introduction & $1-6$ \\
\hline II & Paper 1 & $7-17$ \\
\hline III & Paper 2 & $18-29$ \\
IV & Paper 3 & $30-44$ \\
\hline V & Summary & $45-51$ \\
\hline VI & References & $52-55$ \\
\hline VII & Table & 56
\end{tabular}




\section{Introduction}

\section{A. Background Literature Review}

\section{A.1 Work-Related Musculoskeletal Disorders in Healthcare Workers}

Healthcare workers in the United States experience one of the highest injury rates among all industries. ${ }^{1}$ The most recent Bureau of Labor Statistics (BLS) data in 2018 reported an injury rate of 8.1 injuries per 100 full time hospital workers (FTE). ${ }^{2}$ This places it in the top 15 industries with highest reported worker injuries. These work related injuries affect an employee's ability to work and safely care for patients.

Many of these injuries are related to patient handling and mobilization. ${ }^{3-5}$ This is especially true for those who regularly perform patient mobility tasks, including nurses and rehabilitation professionals. ${ }^{4,6-11}$ These tasks include repetitive lifting (moving and repositioning) as well as the inadvertent use of sustained or repetitive awkward posturing during these activities. This, combined with the increasing size and dependency levels of patients, aging of both employees and patients, and decreased staffing levels within inpatient facilities, all place healthcare workers at a high risk of musculoskeletal injuries. ${ }^{4,6,8,9}$

Though less studied than other healthcare professions, several recent studies have quantified work-related injuries among rehabilitation professionals (Physical and Occupational Therapists and Assistants). ${ }^{4,6}$ In a prospective cohort study of Physical Therapists nationally, Campo et al found a one year incidence rate of new musculoskeletal disorders of $20.7 \%$ and a prevalence of musculoskeletal disorders of $57.5 \% .{ }^{4}$ In a regional survey of 1,189 Physical and Occupational Therapists, Darragh et al found an incident rate of 17/100 Full Time Equivalents (FTEs). ${ }^{6}$ The most recent data available from the Bureau of Labor Statistics (BLS) Survey of Occupational Injuries and Illnesses (SOII) shows that in 2018 there were 610 reported injuries among Physical Therapists and 630 injuries among Occupational Therapists for an injury rate of 32/100,000 FTE for Physical Therapists and 77.2/100,000 FTE for Occupational Therapists. ${ }^{1,12}$

These studies also quantified the association between the job-related tasks and work-related musculoskeletal injuries. Campo et al found that physical therapists who transferred patients six to ten times per day were 2.4 times more likely to develop low back pain when compared to physical therapists who did not perform transfer activities as part of their patient treatment. Additionally, physical therapists who repositioned patients in bed more than ten times per day were 2.6 times more likely to develop low back pain. In addition to work-related exposures, postures or positions during these exposures nearly doubled the risk. Those who reported exposure to bent or twisting postures had 5.7 times higher odds of developing any type of work-related musculoskeletal disorders than those who did not report exposure to these postures. ${ }^{4}$ Similar exposures and resultant work-related injuries have been described in the nursing profession as well. A survey of 1,396 hospital nurses in Portugal found that those who performed any task repetitively more than 10 times per day demonstrated an increased risk of musculoskeletal pain (OR $=2.5$ for repositioning in bed and 2.2 for 
patient feeding in bed). ${ }^{10}$ Therefore, as can be seen, healthcare workers exposed to frequent patient mobility tasks are at risk for developing work-related pain.

\section{A.2 Description and Development of SPHM Programs}

The alarming results of these work-related studies performed over the past couple of decades have forced the healthcare industry to rethink its approach to patient mobility tasks through the creation and implementation of Safe Patient Handling and Mobility (SPHM) programs. Ultimately, these programs ask the healthcare industry to rethink its approach to patient mobility. These programs were pioneered by the Veterans Administration Health System, the National Institute of Occupational Safety and Health (NIOSH), and the American Nursing Association. ${ }^{13-16}$ There are several guiding documents created by these constituents on the creation and execution of an SPHM program. These documents state that these programs should be comprehensive in nature and include interventions at each of the three levels of engineering, administrative and personal/behavioral controls in order to be successful. ${ }^{13-18}$ This includes the purchasing and ongoing care of the appropriate types and amounts of mechanical lift equipment, adoption of policies regarding the use of these devices, and support for employees using the devices via the establishment of ongoing training programs and program leaders. ${ }^{14}$

\section{A.3 Effectiveness of SPHM Programs on Reducing Work-Related Musculoskeletal Disorders}

Multiple research studies have now demonstrated that the use of mechanical lift equipment by health care workers reduces work-related demands and musculoskeletal disorders. ${ }^{19-25}$ One of the earliest studies to report on the effectiveness of these programs was a randomized controlled trial by Yassi et al. In that study, all healthcare professionals that performed patient mobility related tasks were randomized to one of three groups. The three arms of this randomized trial included a control group in which normal patient mobility practices were maintained, a group in which minimal equipment was provided for use for "appropriate" patients, and a third group in which a "no lift" policy was implemented and extensive equipment provided. Although the differences in injury rates among the groups were not statistically significant there were significant findings with regards to increased comfort and decreased work demand among those in the "no lift" group. ${ }^{25}$

Evanoff et al studied the effects of mechanical lifts on worker injury rates and lost work time among nursing personnel in both hospitals and long-term care facilities. This pre-post intervention study examined the differences in recorded injuries via OSHA logs and lost time from work per human resources data to conclude that both demonstrated a statistically significant decline post-intervention. ${ }^{23} \mathrm{~A}$ similar study within a small community hospital conducted pre and post SPHM intervention surveys among nursing personnel. Although the sample size was relatively small, (36 completed both surveys) they found statistically significant improvements in musculoskeletal comfort postintervention. They also analyzed OSHA logs, workers compensation data and hospital payroll data during the study period and reported decreased lost injury days and workers compensation costs following program intervention. ${ }^{22}$ 
Yoder et al reported on the outcomes following implementation of a Safe Patient Handling Program at a large teaching hospital in 2013. Comparing pre-post intervention employee accident reports, workers' compensation records and OSHA logs, they found a reduction in work-related musculoskeletal disorders among all employees involved in patient mobility. They were also able to describe the location and source of many of these injuries due to patient mobility tasks. Prior to program implementation, they found that greater than $70 \%$ occurred in the hospital (outpatient clinics within the hospital were also considered) and that $68 \%$ occurred during transferring or repositioning the patient in bed. Post-intervention these numbers decreased to $35 \%$ and $38 \%$ respectively. It should be noted that only descriptive statistics were reported for this pre-post study. ${ }^{24}$

While establishing the overall effectiveness of SPHM programs on reducing the incidence of WMSDs as well as injury-related costs, these studies either focus exclusively on nursing personnel or analyze all healthcare workers who perform patientmobility tasks without differentiating by occupation. Several recent systematic reviews have pointed out this gap. Mayeda-Letourneau, in a recent critical review of the SPHM literature found that most of this literature has focused on nursing personnel, as they comprise a large majority of the patient mobility staff. ${ }^{20} \mathrm{~A}$ recent systematic review by Harwood et al stated that little could be concluded about the specific effects of SPHM programs on rehabilitation professionals injury rates due to the low quality of available studies, the small number of physical and occupational therapists in the study and the lack of specific injury data. They concluded this review by stating that, "the effects of SPHM programs on rehabilitation practitioner injury rates have not been systematically assessed at this point. Therefore, research focused on rehabilitation professionals engagement with SPHM programs is needed." ${ }^{66}$

The effectiveness of these comprehensive SPHM programs in reducing workrelated musculoskeletal injuries and associated costs has been established. However, as will be discussed in greater detail below, these studies are done primarily with nurses or on undifferentiated groups of healthcare workers involved in patient handling and SPHM programs. Thus, many questions remain to be answered regarding the specific needs, perceptions, and practices of other healthcare professionals, namely rehabilitation professionals, as they participate in these SPHM programs. This dissertation is an attempt to address some of these unanswered questions.

\section{B. Needs and Uses of SPHM Program Equipment Among Rehabilitation Professionals (Paper 1)}

Broadly, perceptions of those who participate in SPHM programs hold positive views of these programs. ${ }^{27-29}$ Healthcare providers that participate in programs that are appropriately implemented and continuously maintained understand the relative value of these programs with regards to patient mobility tasks. Several recent works have explored rehabilitation professional perceptions of and participation within SPHM programs. ${ }^{27-31}$ Findings have included an overall positive perceptions of these programs among the therapists who practice where SPHM programs are present and that the majority of physical therapists used the SPHM equipment to improve patient and worker safety as well as the quality of their interventions. ${ }^{29}$ 
Darragh et al implemented a qualitative focus group approach that included perceptions among rehabilitation professionals on how the equipment is used and its effects on rehabilitation. They found that patient weight, level of function, physical status, psychological status (especially fear and anxiety), presence of wounds, or patients connected to medical lines affected equipment choice and use. They found that the equipment was used most commonly for functional mobility tasks such as bed mobility, transfer training and gait training. Time was reported as both a facilitator and barrier to use. Physical and occupational therapists also reported that this equipment did increase options for mobility therapeutically. Therapists were able to mobilize larger, more dependent patients earlier and provide overall earlier mobility for all patients. They also felt that both themselves and the patients were safer when using the equipment. ${ }^{28}$

However, the specific goals and mechanics used by rehabilitation professionals to execute these patient mobility tasks safely have not been fully explored and may differ from those of other healthcare professionals. They may require longer periods of time or greater repetitions to execute, as such tasks are a central aspect of their patient care plan. ${ }^{5,32}$ Recent studies have called for this in-depth exploration of this aspect of rehabilitation professionals' participation in SPHM programs. Olkowski and Stolfi suggested advancing the research in this area to investigate specifically how the equipment is used and to which patient types it is most commonly applied. ${ }^{29}$ Harwood et al also called for similar work with regards to information on the specific patient care setting, patient level of assistance required, and concurrent mobility tasks being performed in order to more specifically identify appropriate and effective use of mobility lift equipment among rehabilitation professionals. ${ }^{26} \mathrm{~A}$ very recent study by Rugs et al explored this need by having rehabilitation professionals provide photo narratives of their use of lift equipment during the rehabilitation process. The results were across various practice settings and provided early insight but further work in this area is needed. ${ }^{33}$

Therefore, the purpose of this first qualitative research paper was to explore the distinct perceptions, uses, and needs of these work-related programs on rehabilitation professionals. An in-depth qualitative study will allow a more robust description and understanding of their choices and practices when it comes to using mechanical lift equipment. We compare these findings with existing research on the nursing population whenever possible in order to bring light to the distinct needs and practices among rehabilitation professionals.

\section{The Distinct Patient Mobility and Work-Related Injury Experiences Among Rehabilitation Professionals (Paper 2)}

Despite the similar goal of safe patient mobilization, there are some distinct differences in the goals and frequency of the patient mobilization tasks performed between nurses and rehabilitation professionals. Waters and Rockefeller point out that even though the central idea of patient mobility remains the same, the specific tasks and mechanics used by rehabilitation professionals when mobilizing patients differs from other professionals. ${ }^{32}$ These tasks may require longer periods of time or greater repetitions to execute, as it is the central aspect of their patient care plan. 
Given that the specific tasks and work-related exposures are different, the workrelated injury experiences may also differ within the rehabilitation group when compared to other healthcare professions. Most existing studies on injuries due to patient mobilization focus solely on nurses or do not differentiate occupational groups. In the only study found comparing work-related musculoskeletal complaints between nurses and rehabilitation professionals, Alperovitch-Najenson et al, conducted a cross-sectional survey among nurses and physical therapists in a rehabilitation hospital. ${ }^{5}$ They found a statistically significant difference in the prevalence of low back pain in physical therapists (73.1\%) versus nurses (43.9\%) even after adjusting for age, BMI and morbidity. It was also discovered that physical therapists performed full and partial manual patient transfers and walking activities more frequently as well as adopted positions of trunk bending more commonly.

However, previous work has suggested that the culture of independence and pride in physical work has created a reluctance among rehab professionals to acknowledge or report work-related injuries. 6,34,35 Cromie reported nearly 20 years ago that the idea of being seen as capable and hard-working may interfere with the recognition and reporting of injuries among Physical Therapists. This article went on to conclude that, "having a high level of knowledge and skill is valued by the profession and one of the ways of demonstrating this is to remain uninjured." 34 Similar findings were discussed in more recent work by Myers et al that explored the role of culture in work practices among nurses and rehab professionals. They found that the central value among rehab professionals was independence and a pride in their ability to perform lifting tasks safer than nurses. 35

Therefore, the purpose of this second qualitative research paper was to describe the unique patient mobility tasks and work-related injury experiences among rehabilitation professionals. We describe these both generally and within an existing Safe Patient Handling and Mobility (SPHM) program. We compare the findings of our study with existing research studies on the nursing population whenever possible in order to bring light to the distinct needs and practices among rehabilitation professionals.

\section{Effects of Hospital Organization and Administration on Rehabilitation Professionals Involvement in SPHM Programs (Paper 3)}

The use and effects of this lift equipment has been readily studied and found to decrease the risk of work-related injuries among these work groups. ${ }^{20,21,24,25,36}$ However, the organizational and administrative components of these SPHM programs and their effects on healthcare workers are much less reported. In his exploration of patient safety in healthcare, Dekker discussed the idea that healthcare is one of the only industries with a central focus on safety to resist the ideas of systems approaches and theories. He states, "healthcare has a strong preoccupation with individuality." ${ }^{37} \mathrm{He}$ goes on to discuss how this focus solely on the individual interactions among healthcare practitioners and patients misses the numerous other factors that result in poor employee and patient outcomes. The Human Factors Approach to Ergonomics that he describes proposes that we take a broader look at the circumstances of the healthcare 
system and determine how we could redesign the system to make human error less likely. This is especially applicable to comprehensive, system-wide programs such as SPHM programs. Without a broader explanation of the system in which these programs are employed, their successes and failures are not fully explained and, more importantly, their ability to be maximally effective and sustainable are missed.

In a comprehensive study looking at SPHM program implementation at a large teaching hospital in North Carolina, Schoenfisch et al state that we must look at factors beyond just quantitative employee injury counts and rates as measures of successes for these programs. ${ }^{38}$ Through an in-depth qualitative exploration of focus groups and interviews, they found that factors such as unit and room layouts, storage spaces and processes, and other organizational factors might affect the successes or potential failures of these programs. They suggest it is crucial for further research to include the contextual information of the organization in which the program is being implemented in order to maximize program understanding and effectiveness.

Additionally, many of the current systematic reviews on workplace injury reduction programs are inconclusive or deemed not effective as most studies focus primarily on the work and workers and do not explore the effect of the organizational practices and policies. ${ }^{39-41}$ Several recent studies have demonstrated the effectiveness of including and exploring organizational policies and practices on various aspects of workplace injuries. Tveito et al explored the impact of organizational policies and procedures in the hospital setting and found that injury rates decreased in units where nurses reported positive or higher perceptions of the organizational policies and procedures. ${ }^{42}$.

Therefore, the purpose of this third qualitative research paper was to explore the importance of the organizational context and administrative policies on the effectiveness of an SPHM program among rehabilitation professionals. This type of in-depth look at the organizational effects on these SPHM programs has been minimally reported. A deeper look at the organizational policies, beliefs, attention and resources regarding these programs as well as the differences among organizational subgroups will allow for a deeper exploration behind program failures and successes. 


\section{Paper 1}

\section{Abstract: $(288 / 300$ words)}

Objective -The distinct occupational safety and health experiences and needs of rehabilitation professionals (Physical and Occupational Therapists and Assistants) participating in Safe Patient Handling and Mobility (SPHM) programs have not been widely studied. We sought to explore in depth the perceptions, participation, and needs pertaining to lift equipment usage among rehabilitation professionals at a large level-one trauma hospital.

Methods - A comprehensive, ongoing SPHM program was initiated at this facility three years prior to the start of this study. In order to evaluate rehabilitation professionals involvement in this program, six focus groups were conducted with 25 members of the rehabilitation team (11 Physical Therapists, 11 Occupational Therapists and 3 Physical Therapist Assistants). Focus groups were recorded and de-identified transcripts were analyzed for emergent themes.

Results - Several primary themes emerged from the focus groups including uses and needs of SPHM program equipment unique to rehabilitation professionals, notably the common application of some lift equipment for rehabilitation purposes as well as recommendations for equipment modifications to improve its therapeutic value. Less important to the study participants was the use of lift equipment as instruments to improve their own workplace safety.

Conclusions - Rehabilitation professionals in this study are supportive of and interact daily with the SPHM program. However, they often default to manual mobility techniques. This may be partially due to equipment incompatibility with the mobility and functional needs of their patients. Participants in this study provided rich and consistent descriptions for equipment modifications to improve use and applicability during rehabilitation.

Impact - To maximize participation of rehabilitation professionals in SPHM programs, modifications to lift equipment should be considered. These changes will serve to improve patient and practitioner safety as well as patient mobility within these acute care settings. 
Introduction: (4454/4500 words)

Though less studied than other healthcare professions, several recent studies have quantified work-related injuries among rehabilitation professionals (Physical and Occupational Therapists and Assistants). ${ }^{4,6}$ In a prospective cohort study of Physical Therapists nationally, Campo et al found a one year incidence rate of new musculoskeletal disorders of $20.7 \%$ and a prevalence of musculoskeletal disorders of $57.5 \% .{ }^{4}$ In a regional survey of 1,189 Physical and Occupational Therapists, Darragh et al found an incident rate of 17/100 Full Time Equivalents (FTEs). ${ }^{6}$ The most recent data available from the Bureau of Labor Statistics (BLS) Survey of Occupational Injuries and Illnesses (SOII) shows that in 2018 there were 610 reported injuries among Physical Therapists and 630 injuries among Occupational Therapists for an injury rate of 32/100,000 FTE for Physical Therapists and 77.2/100,000 FTE for Occupational Therapists. ${ }^{1,12}$

Many of these injuries are related to patient mobilization. ${ }^{3-5}$ This is especially true for those who regularly perform patient mobility tasks. ${ }^{4,6-11}$ These tasks include repetitive lifting (moving and repositioning) as well as the inadvertent use of sustained or repetitive awkward posturing during these activities. This, combined with the increasing size and dependency levels of patients, aging of both employees and patients, and decreased staffing levels within inpatient facilities, all place healthcare workers at a high risk of musculoskeletal injuries. ${ }^{4,6,8,9}$

To address this problem, various inpatient facilities have implemented Safe Patient Handling and Mobility (SPHM) programs. These programs are comprehensive in nature and include interventions at each of the three levels of engineering, administrative, and personal/behavioral controls. ${ }^{13-18}$ This includes the purchasing and ongoing care of the appropriate types and amounts of lift equipment, adoption of policies regarding the usage of these devices, and support for employees using the devices via the establishment of ongoing training and program leaders. ${ }^{17}$ Rehabilitation professionals perform patient mobility tasks daily as a central part of their scope of practice, especially within inpatient facilities where these SPHM programs exist. However, the specific goals and mechanics used to execute these patient mobility tasks may differ from other healthcare professionals. They may require longer periods of time or greater repetitions to execute, as such tasks are a central aspect of their patient care plan. 5,32 Thus, the specific needs and applications of these programs among rehabilitation professionals should be explored. This will begin to allow these comprehensive programs to be tailored to the specific needs of this understudied occupational group and will improve adoption and safety measures for both patients and practitioners alike.

Recent studies have called for this in-depth exploration of this aspect of rehabilitation professionals' participation in SPHM programs. Olkowski and Stolfi suggested advancing the research in this area to investigate specifically how the equipment is used and to which patient types it is most commonly applied. ${ }^{29}$ Harwood et al also called for similar work with regards to information on the specific patient care setting, patient level of assistance required, and concurrent mobility tasks being performed in order to more specifically identify appropriate and effective use of mobility 
lift equipment among rehabilitation professionals. ${ }^{26}$ A very recent study by Rugs et al addressed this need by having rehabilitation professionals provide photo narratives of their use of lift equipment during the rehabilitation process. The results were across various practice settings and provided early insight but further work in this area is needed. ${ }^{33}$

Therefore, the purpose of this qualitative research project was to explore the distinct occupational safety and health experiences of rehabilitation professionals who participate in SPHM programs. More specifically, we sought an improved understanding of the perceptions, participation, and needs of these work-related programs on this distinct employee subgroup. We compare these findings with existing research on the nursing population whenever possible in order to bring light to the distinct needs and uses among rehabilitation professionals.

\section{Methods}

\section{Research Design}

A qualitative case study design was employed for this study given that the primary purpose was to provide an in-depth description and analysis of a bounded system. ${ }^{43,44}$ In this study, the bounded system was an SPHM program. This approach allows researchers to study complex phenomena in their natural setting and seeks to provide a rich, holistic description of the issues from the perspective of participants. ${ }^{43,44}$ The cases, or participants, are selected based on the study purpose and what they can reveal about the system or topic of interest.

\section{Study Site and Program Description}

The study site was a nearly 700 bed academic medical center with a hospitalwide SPHM program established in 2015. The SPHM program included a multilevel implementation strategy that consists of mechanical lift equipment, policy changes for patient lifting, and establishment of program trainings and lift team meetings. The planning and establishment of this program was led by the hospitals safety department. The equipment purchased included equipment that could assist with the performance of supine to sit transfers, mechanical and manual standing and raising aids, lateral transfers and repositioning devices, floor lifts, and ceiling lifts. ${ }^{45}$

\section{Investigator Perspectives}

The team of investigators included two Physical Therapists (one with an academic doctorate in Occupational Health with extensive research experience within this field), three occupational health research scientists (one with extensive research experience within this field and within similar SPHM programs), and an occupational medicine physician. This diversity of backgrounds helped enrich the perspectives on the study findings. The research team often discussed and recognized their own contributions and biases during the research process.

\section{Sampling Strategy and Data Collection}


Due to the qualitative methodology of this study, purposeful sampling methods were employed. ${ }^{44}$ This approach intentionally samples a group of people that can best inform the researcher about the research problem under examination. ${ }^{44}$ Study participants recruited for the study were Physical and Occupational Therapists and Assistants who performed patient mobility tasks throughout a majority of their day. Recruitment occurred through the study site gatekeepers via emails (including a recruitment flyer) and word of mouth. Interested participants emailed the primary study investigator and initially earned a $\$ 20$ gift card for their involvement that was later increased to $\$ 140$ in gift cards to increase participation. This study was approved by the Institutional Review Board and informed consent was obtained before the start of each focus group.

Focus groups were chosen as the primary method for data collection. The advantages of focus groups are that they allow for participant discussion and interactions. This is particularly beneficial for topics, such as this SPHM program, that could be discussed comfortably in everyday life. ${ }^{43}$ Focus groups consisted of various combinations of rehabilitation professionals (Physical and Occupational Therapists and Assistants) and were scheduled and structured based on participant availability. Each focus group lasted between 60 and 90 minutes and were guided by semi-structured guides. The guide was used to ensure discussion of work tasks and procedures, SPHM program experiences, equipment use, equipment needs, and barriers to equipment use. Focus groups were initially held in person, however, the COVID-19 pandemic forced the remaining three focus groups to occur via Zoom. In-person and Zoom focus groups were recorded using digital voice recorders or the built-in Zoom recording feature. Participants used alias names during the focus group process to ensure anonymity. The primary investigator also took field notes during each focus group. The data was transcribed using the human transcription option through Rev.com. ${ }^{46}$

\section{Data Analysis Procedures}

Content or thematic coding was used to analyze the results. This approach allowed us to identify common feedback and establish a "framework of thematic ideas" as described by Gibbs. ${ }^{47}$ The primary and secondary study investigators independently analyzed the transcribed data using first and second cycle coding procedures. ${ }^{48}$ First cycle coding procedures included attribute coding, descriptive coding (topic based) and in vivo coding (terms used by participants themselves) initially using a sentence by sentence initial coding process and then holistic paragraph by paragraph coding to develop open code categories. ${ }^{48}$ First cycle coding was performed by hand and then uploaded to Microsoft Office Word for organization and secondary coding and analysis.

\section{Results}

\section{Study Participants}

Twenty-five rehabilitation professionals (11 Physical Therapists 11 Occupational Therapists, and 3 Physical Therapist Assistants) participated in six focus groups over a nine-month period. Focus groups included between three and eight participants. The 
sample comprised $49 \%$ of the Rehabilitation Department at the study site. The years of work experience among participants ranged from 7 months to 14.5 years and their overall time from graduation ranged from three to 26 years. All but one of the participants were female (See Table).

\section{Themes}

Four primary themes emerged following data analysis. These included SPHM program support, judgements for lift equipment usage, lift equipment preferences, and lift equipment changes for rehabilitation needs. These themes were centered around the goal of therapeutic patient mobility.

\section{$\underline{\text { SPHM Program Support }}$}

Overall, participants were positive towards the SPHM program. Of the 25 participants, none indicated they were not in favor of the SPHM program. They thought it helped improve their ability as rehabilitation professionals to mobilize patients that they may not otherwise be able to mobilize or transfer.

"I think the lift equipment just helps in general. I think it helps us to be better clinicians, and I think it helps the patients just getting them moving around more."

“....and I also think if I'm alone and I don't have another pair of hands, I can stand or transfer people alone with the [manual standing and raising aid] that I wouldn't dream of standing or transferring alone."

“...but I think six or seven years ago before this stuff came around, we probably would have just laid him back down. And I like that we can pull in the [manual standing and raising aid] and then be able to get them to the sink."

\section{Judgements for Lift Equipment Usage}

Participants noted they considered various patient characteristics when deciding to use the lift equipment that they continue to rely heavily on manual transfer techniques and that using the lift equipment may decrease injury risk for themselves or their patients. In terms of patient characteristics, participants described the equipment as ideal for those with generalized weakness and deconditioning, and for those with cognitive impairments, fear or anxiety.

"Just deconditioned geriatrics, like a nursing home resident who normally does pivots to their wheelchair but they're just acutely deconditioned."

"I mean, the [manual standing and raising aid] is good for gross weakness patients."

"It was with a cognitively impaired patient, so they weren't able to follow our commands and our queuing to safely transfer side to side to get to a chair. So it was easier to use the [manual standing and raising aid]. For some reason, when 
you put that piece of equipment in front of them and have them just put their hands up, they just automatically know how to pull."

"If you try the [manual standing and raising aid]...they feel safer as well. They might have high anxiety or need something to make them feel more secure...like it's just a tool that we can use for safety until they are able to do it in a safe manner."

Another key subtheme regarding clinical judgements is the preference among participants to try a manual transfer prior to using the mechanical lift equipment. This was consistently heard across all focus groups. In terms of when they are choosing to use the equipment in the rehabilitation process, it was overwhelmingly reported that they used the equipment when a manual transfer failed or felt unsafe.

"I always usually at least try either a sit to stand or a modified stand pivot transfer first, before I will get a [manual standing and raising aid] or something like that. So usually on my eval, I tend to see how they do without any equipment and then maybe incorporate some of that in the treatment next time."

"If we do about two or three attempts to stand and it goes nowhere, that's the [manual standing and raising aid]."

"If I had to choose between using a [manual standing and raising aid] and doing an actual transfer to work on it, I'm going to do a transfer over using the [manual standing and raising aid] as well."

Some participants reported that decreasing the chance of injury has also started to play into their decision to use equipment. They acknowledge the risk of injury without the equipment and report that sometimes if they feel they are in a situation where pain or injury could occur, they will choose to use the equipment.

"I think it [the equipment] is just a huge saver of injuries personally."

"I mean I'll weigh that if I have a patient that I'm like, could I hurt them? Could I hurt myself? Then I might take a step back and consider more equipment."

"...there are a lot of times where we go to attempt something and almost immediately think...oh nope I better go grab that piece of equipment [or I will get hurt]."

\section{Lift Equipment Preferences}

Unequivocally, the manual standing and raising aid consistently rose to the top as the most used piece of equipment among participants due to its ease of use and availability on most units.

"...[manual standing and raising aid] ...That's probably my favorite piece of equipment to help get somebody out of bed." 
"I think the [manual standing and raising aid] lets us do many things quick."

"And it's [manual standing and raising aid] available on every floor. There's no sling."

Another point regarding the manual standing and raising aid was that it allowed for therapeutic mobility, which was described as promoting active patient participation. Many also cited its ability to be used during various functional tasks including transfers, toileting, and grooming activities.

"I think it's good to note that we use the [manual standing and raising aid] ...I think it's all of our favorites because we use it as a therapy tool."

"Like somebody can be up at a sink, somebody can be standing in front of their closet. Somebody can actually be mobilized into a real bathroom, which is also meaningful and private as opposed to having to use a bedside toilet. So the functionally the [manual standing and raising aid] is OT friendly and rehab friendly."

"So I feel like using it [manual standing and raising aid] as a therapeutic tool just within our therapy session is a huge use of our equipment as well."

The second most commonly used piece of equipment, the floor lift, was reported to be more user friendly than other versions of dependent floor lifts and was noted to be commonly recommended to nursing staff.

"I've used the [floor lift] a handful of times and it's nice. It's a little easier to work with than a regular Hoyer that l've used in the past."

"I often make the suggestion of, 'You can always use the [floor lift]', to nursing."

The least frequently used pieces of equipment among this group of rehabilitation professionals were the mechanical standing and raising aid, the ambulatory device and the lateral or repositioning slides. The mechanical standing and raising aid was reported in most focus groups to be used the least of all equipment. Participants reported the sling not fitting most patients and that it does not encourage a normal sit to stand movement pattern (patients tend to lean back instead of forward in it).

"It's not like the movement pattern that the [mechanical standing and raising aid] gives you is not the movement pattern that you would teach somebody to transfer."

"The weight shift isn't there like it...so they end up...leaning back."

"I think they're [mechanical standing and raising aid] really awkward...like the sling always slides up... and they're really uncomfortable." 
The ambulatory device was not reported to be readily available on the floors (only one piece located in the rehab gym) and was described as bulky and time consuming to use.

"I really want to use it [the ambulatory device] somewhere so badly. It's just the length of stay is so short...it's also super time consuming"

"We only have one right here in our department."

"Yeah and it's bulky [the ambulatory device]. So it is a little harder to manage."

Lateral or repositioning slides were not reported as frequently used. Participants were not sure where they were kept and also reported they were too time consuming to place. They reported they were used primarily for bed to mat transfers in the rehab gym. When repositioning patients in their hospital bed, they still report using draw sheets to do so.

"I don't even know where we keep them [repositioning slides] to be honest."

"I think it takes too much time to put it under them [repositioning slides] and they would just put them in trendelenberg or get an extra person because it's quicker I guess."

"...whenever we have patients in our rehab department...we use those [repositioning slides] to transfer patients from the beds to the mat table. So it is used with almost every patient that we do that with here at the rehab gym."

"We just use a draw sheet for a bed move. With our technique and proper body mechanics, typically a draw sheet is all we need. I don't feel like we actually need the [repositioning slides]."

However, a couple of participants did report that they felt like the repositioning slides were underutilized within this facility.

"We use the slides a lot in the rehab gym whenever we're doing a mat table with patients. Or if you're up on the floor and you're getting a patient up to a shuttle chair or something like that. But we don't use them a whole lot, and I feel like they're definitely way underutilized in the facility as a whole."

"They're great thought for a patient who, like on the mat table, a patient who's really big and hard to turn with the draw sheet. But I think you're right. I think we don't use them enough."

\section{Lift Equipment Improvements for Rehabilitation Needs}

Another theme centered on the need for equipment changes to better meet rehabilitation needs. Participants had specific suggestions for improvements to the manual standing and raising aid (manual standing and raising aid) that included a larger 
weight limit (or bariatric version), a wider area for the hips, and an adjustable knee and footplate. The adjustable knee plate was suggested to accommodate different patient heights. An adjustable footplate that could be completely removed on one side was also suggested to accommodate an amputation or limited weight bearing.

"So sometimes I have a larger patient who I can't use the [manual standing and raising aid] with. That's been a barrier that I have seen, and it's been difficult to mobilize a larger patient compared to an average patient."

"My favorite one, the [manual standing and raising aid], because it's the most utilized...but again, with the hip width, sometimes it's just not wide enough. And the weight rating on the [manual standing and raising aid] isn't that high."

"I would like to see a [manual standing and raising aid] with a knee plate that adjusts. That would be really nice, because l've had a lot of taller individuals and then it's just at an awkward place that hits too low on them."

"To have one [foot plate] that you can move to one side versus the other. For instance, our weight bearing restrictions, if they're not weight bearing on one leg it would be lovely to just to move the [foot] plate."

\section{Discussion}

The results of our study demonstrate broadly supportive attitudes towards SPHM programs among rehabilitation professionals. While there were suggestions for improvements, most participants saw the importance of the SPHM program and lift equipment in the hospital-based rehabilitation process. This is consistent with several recent studies that demonstrate overall support of SPHM programs among physical and occupational therapists. Olkowski and Stolfi, in a nationwide survey of Physical Therapists, found that those who practice in an inpatient environment where SPHM programs are present demonstrate overall positive perceptions of these programs. ${ }^{29}$ Similar sentiments were echoed in a comparable qualitative study by Darragh, et al, in which physical and occupational therapists viewed they these SPHM programs as "generally helpful". ${ }^{27}$

However, even though participants were supportive of SPHM programs, they routinely defaulted to manual transfer techniques. This was consistently heard across all focus groups. Participants indicated that they chose to use the equipment only under certain circumstances including when a manual transfer failed or felt unsafe, during follow up treatments more than initial evaluations, and when they did not have an extra set of trained hands for assistance.

Clinical decision-making pertaining to the use of lift equipment was discussed by participants in terms of three main factors - patient characteristics, transfer type, and work safety. Clinical decision-making was described in a recent concept analysis in Physical Therapy Journal was defined as "integrating cognitive, psychomotor, and affective skills. It is contextual in nature and involves both therapist and client 
perspectives. It is adaptive, iterative, and collaborative with the intended outcome being a biopsychosocial approach to patient/client management." 49

In terms of clinical decision-making based on patient characteristics, participants in this study described the equipment as ideal for those with generalized weakness and deconditioning, and for those with cognitive impairments, fear or anxiety. Research in this area among rehabilitation professionals is variable. A similar study with rehabilitation professionals found that greater patient size and lower level of function increased chance of equipment usage. ${ }^{27}$ Additionally, it has been reported that larger physical status, psychological status (especially fear and anxiety), and presence of wounds or lines affected equipment choice and use. ${ }^{28}$

Comparable themes are found in the nursing literature regarding lift equipment decision-making based on patient characteristics. Similar to our study, the presence of lines and tubes have been reported as a barrier to equipment use among nurses. ${ }^{50}$ Alternatively, increased patient fear and anxiety were reported to decrease equipment usage. ${ }^{50}$ Additional research is warranted among both professions regarding specific patient characteristics driving lift equipment usage.

Some participants reported that decreasing the chance of injury has begun to factor into their decision to use the lift equipment. They acknowledge the risk of injury without the equipment and report that sometimes if they feel they are in a situation where pain or injury could occur, they will choose to use the equipment. The realization of risk of injury with patient mobility overall among therapists has been discovered in previous studies but has only been more recently discussed as a reason for lift equipment usage. , 28 Similar themes are starting to emerge in the nursing literature as well with a recent qualitative study finding concern for personal health as a motivator for lift equipment usage. ${ }^{51}$

One of the most robust areas of discussion for participants in this project centered around the type of lift equipment used. Participants identified equipment they frequently used, equipment they infrequently used, and offered suggestions for equipment modifications to increase the use of the equipment as well as improve its therapeutic value. Unequivocally, the manual standing and raising aid was the piece of equipment used most by rehabilitation professionals within this study. The common reasons behind the popularity of this equipment included ease of use, availability and ability to be used in a therapeutic manner.

This idea of using this piece of lift equipment therapeutically was important to participants as improving patient mobility and function was seen as a central role of their work. Participants cited specific tasks that they could complete using the manual standing and raising aid such as toileting, sink activities and prolonged standing that may have not otherwise been possible. Similar studies among rehab professionals have also reported the manual standing and raising aid as the most frequently used piece of equipment and that using the equipment therapeutically for transfer and gait training is very common. ${ }^{27,28}$ 
Studies among nurses are finding different patterns of equipment usage than we observed among our study participants. A recent survey among nurses and nurse assistants within a similar academic medical center found that the dependent floor lift and the mechanical sit-to-stand lift were the most commonly used pieces of equipment among that work group. ${ }^{50}$ Another study that discusses similar items among nursing professionals found that about $60 \%$ of survey respondents did not use these devices during functional tasks such as transferring patients out of bed, to a bedside commode or to the bathroom. ${ }^{30}$ This contrast in findings is indicative of the difference in focus and scope of practice between nurses and rehabilitation professionals.

One area of this study that, to our knowledge, has not been previously described includes suggestions for equipment modifications for rehabilitation needs. These came up prominently within each focus group. These recommendations were for the manual standing and raising aid and included a bariatric version, wider hip area, removable unilateral footplate, and a height adjustable knee plate. These modifications by SPHM program equipment representatives would allow the rehabilitation group to participate more fully in these types of programs and, more importantly, would allow the safer movement of patients for all involved. This information addresses the concern more broadly raised by similar studies of rehabilitation professionals that noted that, "these devices were not designed with the facilitation of independence among patients in mind." 27 This study went on to state that, "the involvement of therapists in the design of lift equipment will be crucial if they are to protect themselves and their patients from injury." ${ }^{27}$ We conclude that these equipment limitations may at least partially explain the continued reliance on manual transfer techniques among rehabilitation professionals.

\section{Limitations}

The primary limitation of this study is the fact that this is a single-site study. Since qualitative case studies are not meant to be generalizable, comparisons must be made carefully and within the context of this setting and comparable settings in mind. In this case, it is worth noting that this setting was a teaching hospital in a small urban community and that comparisons outside this kind of setting are particularly problematic. However, the SPHM program implemented is structured similarly to other SPHM programs nationwide and these findings may, therefore, be transferrable. In addition, our findings are similar to those demonstrated in other studies among rehabilitation professionals. More research at different study sites with varied methodology would help to validate these findings.

In conclusion, the results of this study help to fill a gap in the literature pertaining to rehabilitation professionals' distinct needs and uses of patient lift equipment. This includes the preference of the manual standing and raising aid as the favored piece of equipment and several suggestions for improvements to this equipment to make it more useful for rehabilitation purposes. Additionally, it is important to note that rehabilitation professionals often utilize lift equipment as therapeutic devices and less often as instruments to improve their own workplace safety. Should equipment manufacturers begin to include some of these suggestions into equipment design, it may increase rehabilitation professionals' use of lift equipment during patient mobility tasks. This would lead to improved practitioner safety among this distinct occupational group. 


\section{Paper 2}

Title: A Qualitative Exploration of the Unique Work and Work-Related Injury Experiences among Physical and Occupational Therapists and Assistants Participating in a Safe Patient Handling and Mobility Program

Abstract: (322 words)

Introduction - Research has shown that the patient mobility tasks required in the acute care setting place healthcare workers, including rehabilitation professionals, at a high risk for workrelated musculoskeletal disorders (WMSDs). However, less is known about the specific patient handling risks rehabilitation professionals face. Therefore, we sought to explain the unique patient handling work and injury experiences among rehabilitation professionals.

Methods - A comprehensive, ongoing SPHM program was initiated at this facility three years prior to the start of this study. In order to evaluate rehabilitation professionals involvement in this program, six focus groups were conducted with 25 members of the rehabilitation team (11 Physical Therapists, 11 Occupational Therapists and 3 Physical Therapist Assistants). Focus groups were recorded and de-identified transcripts were analyzed for emergent themes.

Results - Five primary themes emerged following data analysis. Rehabilitation professionals overwhelmingly described patient mobility as the primary aspect of their job. Central to this was the application of patient lift and transfer devices for therapeutic purposes. They prioritized patient involvement in the mobility task. Participants in this study were quick to deny workrelated injuries but frequently described experiencing work-related pain. They rarely reported these bouts of work-related pain to employers but instead chose to self-manage through treatment by co-workers, use of over-the-counter medications, or informal alteration in job tasks. They did perceive that the lift equipment improved their work-related safety.

Conclusion - In order to maximize the safety of this distinct work group, several recommendations for SPHM trainings and future research should be considered. SPHM trainings should include examples of therapeutic use of lift equipment for rehabilitation professionals in order to maximize their participation in SPHM programs. Additionally, when measuring the effect of these SPHM programs on this work group, hospital injury records should not be used but rather information gained directly from the workers. Researchers should be careful to include the terms work-related pain instead of work-related injury when asking about the adverse effects of patient mobility. 
Introduction: (5024 words)

Hospital workers in the United States experience one of the highest injury rates among all industries. ${ }^{1}$ The most recent Bureau of Labor Statistics (BLS) data in 2018 reported an injury rate of 8.1 injuries per 100 full time hospital workers (FTE). ${ }^{2}$ This places it in the top 15 industries with highest reported worker injuries. Though less studied than other healthcare professions, several recent studies have quantified work-related injuries among rehabilitation professionals (Physical and Occupational Therapists and Assistants). ${ }^{4,6}$ In a prospective cohort study of Physical Therapists nationally, Campo et al found a one year incidence rate of new musculoskeletal disorders of $20.7 \%$ and a prevalence of musculoskeletal disorders of $57.5 \% .{ }^{4}$ In a regional survey of 1,189 Physical and Occupational Therapists, Darragh et al found an incident rate of 17/100 Full Time Equivalents (FTEs). ${ }^{6}$

Many of these injuries among healthcare workers, especially in the acute care setting, are related to patient mobilization. ${ }^{3-5}$ This is especially true for nurses, physical therapists and occupational therapists who regularly perform patient mobility tasks. ${ }^{4,6-11}$ These tasks include repetitive lifting (moving and repositioning) as well as the inadvertent use of sustained or repetitive awkward posturing during these activities. This, combined with the increasing size and dependency levels of patients, aging of both employees and patients, and decreased staffing levels within inpatient facilities, all place healthcare workers at a high risk of musculoskeletal injuries. ${ }^{4,6,8,9}$

Despite the similar goal of safe patient mobilization, there are some distinct differences in the goals and frequency of the patient mobilization tasks performed between nurses and rehabilitation professionals. Waters and Rockefeller point out that even though the central idea of patient mobility remains the same, the specific tasks and mechanics used by rehabilitation professionals when mobilizing patients differs from other professionals. ${ }^{32}$ These tasks may require longer periods of time or greater repetitions to execute, as it is the central aspect of their patient care plan.

Given that the specific work tasks are different, the work-related injury experiences may also differ within the rehabilitation group when compared to other healthcare professions. Most existing studies on injuries due to patient mobilization do not differentiate healthcare professions or focus solely on nurses. In the only study found comparing work-related musculoskeletal complaints between nurses and rehabilitation professionals, Alperovitch-Najenson et al, conducted a cross-sectional survey among nurses and physical therapists in a rehabilitation hospital. ${ }^{5}$ They found a statistically significant difference in the prevalence of low back pain in physical therapists $(73.1 \%)$ versus nurses (43.9\%) even after adjusting for age, BMI and morbidity. It was also discovered that physical therapists performed full and partial manual patient transfers and walking activities more frequently as well as adopted positions of trunk bending more commonly.

However, previous work has suggested that the culture of independence and pride in physical work has created a reluctance among rehab professionals to acknowledge or report work-related injuries. ${ }^{6,34,35}$ Cromie reported nearly 20 years ago that the idea of being seen as capable and hard-working may interfere with the recognition and reporting of injuries among 
Physical Therapists. This article went on to conclude that, "having a high level of knowledge and skill is valued by the profession and one of the ways of demonstrating this is to remain uninjured." 34 Similar findings were discussed in more recent work by Myers et al that explored the role of culture in work practices among nurses and rehab professionals. They found that the central value among rehab professionals was independence and a pride in their ability to perform lifting tasks safer than nurses. ${ }^{35}$

Therefore, the purpose of this qualitative research project was to describe the unique work and injury experiences among rehabilitation professionals. More specifically, we describe these both generally and within an existing Safe Patient Handling and Mobility (SPHM) program. These SPHM programs were created in response to the alarming rate of injuries among healthcare workers performing patient mobility tasks. They are comprehensive in nature and include the purchasing of mechanical lift equipment, adoption of policies regarding the use of these devices, and support for employees using the devices ${ }^{13-18}$ We compare the findings of our study with existing research studies on the nursing population whenever possible. These results will allow these comprehensive programs to be tailored to the specific needs of rehabilitation professionals, which will improve adoption and safety measures for this distinct work group.

\section{Methods}

\section{Research Design}

A qualitative case study design was employed for this study given that the primary purpose was to provide an in-depth description and analysis of a bounded system. ${ }^{43,44}$ In this study, the bounded system was an SPHM program. This approach allows researchers to study complex phenomena in their natural setting and seeks to provide a rich, holistic description of the issues from the perspective of participants. ${ }^{43,44}$ The cases, or participants, are selected based on the study purpose and what they can reveal about the system or topic of interest.

\section{Study Site and SPHM Program Description}

The study site was a nearly 700 bed academic medical center with a hospital-wide SPHM program established in 2015. The SPHM program included a multilevel implementation strategy that consists of mechanical lift equipment, policy changes for patient lifting, and establishment of program trainings and lift team meetings. The planning and establishment of this program was led by the hospitals safety department. The equipment purchased included equipment that could assist with the performance of supine to sit transfers, mechanical and manual standing and raising aids, lateral transfers and repositioning devices, floor lifts, and ceiling lifts. ${ }^{45}$

To support the mechanical lift equipment usage, administrative policy changes were simultaneously implemented. These included a comprehensive SPHM policy and procedures manual, the establishment of an SPHM committee and lift champions, and algorithms to help staff make decisions on when to use the equipment versus when it is safe to perform a manually assisted lift. All employees were initially trained on the new equipment prior to program implementation. This training now also occurs when new employees are hired. The employees 
trained are those that will be involved directly or indirectly with equipment usage. Ongoing yearly trainings also now occur for some employees as a refresher course. Additionally, a team has been established with representatives from all involved departments that continues to meet monthly or every other month to discuss program successes and challenges as well as needs for ongoing implementation. The equipment representatives were onsite periodically throughout the first two years of implementation to assist with program launching and are still available for consultation as needed.

\section{Investigator Perspectives}

The team of investigators included two Physical Therapists (one with an academic doctorate in Occupational Health with extensive research experience within this field), three occupational health research scientists (one with extensive research experience within this field and within similar SPHM programs), and an occupational medicine physician. This diversity of backgrounds helped enrich the perspectives on the study findings. The research team often discussed and recognized their own contributions and biases during the research process.

\section{Sampling Strategy}

Due to the qualitative collective case study methodology of this study, purposeful sampling methods were employed. ${ }^{44}$ This purposeful sample intentionally samples a group of people that can best inform the researcher about the research problem under examination. ${ }^{44}$ Study participants recruited for the study were physical and occupational therapists and assistants employed within the Rehabilitation Department at the study site. These rehabilitation professionals must have performed patient mobility tasks throughout a majority of their day in order to qualify for study inclusion. Recruitment occurred through the study site gatekeepers via emails (including a recruitment flyer) and word of mouth by the Rehabilitation Director and Physical Therapy Manager of the Rehabilitation Department. Interested participants emailed the primary study investigator and initially earned a \$20 gift card for their participation that was later increased to $\$ 140$ in gift cards in order to maximize participation.

\section{Data Collection Procedures}

Focus groups were chosen as the primary method for data collection. The advantages of focus groups are that they allow for participant discussion and interactions. ${ }^{43}$ This is particularly beneficial for topics, such as this SPHM program, that could be discussed comfortably in everyday life. ${ }^{43}$ Focus groups consisted of various combinations of rehabilitation professionals (Physical and Occupational Therapists and Assistants) and were scheduled and structured based on participant availability. Each focus group lasted between 60 and 90 minutes and were guided by semi-structured guides. The guide was used to ensure discussion of work tasks and procedures, SPHM program experiences, equipment use, equipment needs, and barriers to equipment use.

Focus groups were initially held in person, however, the COVID-19 pandemic forced the remaining three focus groups to occur via Zoom. In-person and Zoom focus groups were recorded using digital voice recorders or the built-in Zoom recording feature. Participants used 
alias names during the focus group process to ensure anonymity. The primary investigator also took field notes during each focus group. The data was transcribed using the human transcription option through Rev.com. ${ }^{46}$

\section{Data Analysis Procedures}

Content or thematic coding was used to analyze the results. This approach allowed us to identify common feedback and establish a "framework of thematic ideas" as described by Gibbs ${ }^{47}$ The primary and secondary study investigators independently analyzed the transcribed data using first and second cycle coding procedures. ${ }^{48}$ First cycle coding procedures included attribute coding, descriptive coding (topic based) and in vivo coding (terms used by participants themselves) initially using a sentence by sentence initial coding process and then holistic paragraph by paragraph coding to develop open code categories. ${ }^{48}$ First cycle coding was performed by hand and then uploaded to Microsoft Office Word for organization and secondary coding and analysis.

\section{Results}

\section{Study Participants}

Twenty-five rehabilitation professionals (11 Physical Therapists 11 Occupational Therapists, and 3 Physical Therapist Assistants) participated in six focus groups over a ninemonth period. Focus groups included between three and eight participants. The sample comprised $49 \%$ of the Rehabilitation Department at the study site. The years of work experience among participants ranged from 7 months to 14.5 years and their overall time from graduation ranged from three to 26 years. All but one of the participants were female (See Table).

\section{Themes}

Five primary themes emerged following data analysis. These included the central role of patient mobility in rehabilitation work, the description of work-related exposures, working in pain, the positive effect of lift equipment on perceived injury risk, and the common application of selfmanagement measures to treat work-related pain.

\section{Central Role of Patient Mobility in Rehabilitation Work}

When describing their day to day work, participants overwhelmingly described patient mobility as the primary or central aspect of their job. Central to these patient mobility tasks was the idea of a therapeutic value to the mobility.

"PT, that is our job [patient mobility]...our biggest goal is to get people up and out of bed and moving, and then see how much help they need with that...mobility is what we do here."

"The only time I'm not mobilizing the patient is when l'm chart reviewing or documenting." 
"...our goal is how can we help them utilize their skills to be independent and to do the things that are meaningful to them in our session while we're up there?"

"...our goal is not just to get them up...It's to engage, to participate."

Within this description of patient mobility, rehabilitation professionals explicitly illustrated the differences between themselves and nurses. These differences were described from a rehabilitation perspective and included patient involvement in the process and use of mechanically driven mobility techniques.

“...they're [nurses] doing kind of the easiest way to get a patient from $A$ to $B$....and for us, we're trying to get the patient as involved in the movement as possible..."

"We know what we can do as therapists and we can handle higher levels of difficulty with a patient. Whereas we wouldn't want to put nursing in a situation that could end up being risky if they don't have the manual skills to handle it."

"As therapists, we're in a position where we can challenge the patients a little bit more to do stuff..."

"Nurses get the lift training but we have the skills and the knowledge set from being in school to know how to properly use our bodies to help that individual up into the [manual standing and raising aid]."

\section{Description of Work-Related Exposures}

Multiple patient mobility tasks were cited as risky by participants. These included repositioning in bed, bed mobility/supine to sit transfers, and stand pivot transfers. However, there was no consensus on which patient mobility task presented the greatest risk. A couple of participants cited environmental or patient-related factors as part of the risk including crowded or cluttered rooms and patients that are more dependent.

"I would probably say during bed mobility is where I am most at risk and have tweaked my back a couple of times, but nothing serious."

"For me, it would be more during a lateral [stand pivot] transfer...because it's an awkward position to be in anyway, and I feel like you're already in a half squat yourself."

"I think one of the hardest things too, particularly if you're seeing people by yourself, is laying them back down in bed and...getting them positioned back up to the head of the bed."

"Most times I can think of hurting myself-ish is reaching for things in a patient room that is cluttered and/or crowded. 
"I definitely will have patients that require a lot more assistance and maybe more time is spent with them. So, usually the next day we're all pretty sore from that but now enough that it felt like I couldn't do my job safely."

\section{Working in Pain}

When asked about injuries at work, participants were more likely to describe working in pain than experiencing an injury. No participants reported a personal work-related injury that was reported to their manager or that required medical intervention. They also denied serious injuries or having to miss work due to the pain or injury.

"Not injured necessarily...more like a good workout or some soreness."

"Yeah I've certainly had times where I feel sore the next day but not in pain."

"So I personally haven't had... any work related injuries but I definitely feel some strain and pull more days than others."

"I have not been seriously hurt or anything like that. I will say, like if I have some larger patients for like a couple of weeks, I feel it. I can't ever say that I've gotten injured. Luckily."

"I myself have never really been personally injured that I had to go see a doctor or go to employee health or anything at work."

"...so I work in pain a good bit but l've never been out of work because of an injury."

"And I didn't have any significant injuries, per se, from lifting a patient but I could tell whenever I would do something."

The frequency of working in pain varied from several times a year to several times a week. Participants felt overall that the rate of injuries among rehab professionals was low especially when compared to nurses or other professions.

"I feel like l've tweaked my back once or twice."

"Honestly, just a couple of times a year...not very often." [When asked how common it is to work in pain]

"I mean, usually it's like a small part of the day, a couple of days a week maybe at the most."

"Maybe once a week....maybe a handful of times during the week, once or twice." [When asked how common it is to work in pain]

"Ours [rehab injuries] are fairly low." 
"Although I think as a whole we have far fewer injuries than we hear about in other departments. Far fewer injuries than nursing for example."

The Positive Effect of Lift Equipment on Perceived Injury Risk

Unequivocally, all participants reported that the use of the lift equipment during patient mobility minimized the risk of pain or injury. Some felt this occurred more significantly than others. This was especially true with the manual standing and raising aid that was the most commonly used piece of equipment among this study population.

"The more we use the lift equipment, I don't feel as strained or pulled muscles or anything like that."

"I, myself, over the last probably three years have been dealing with some issues of my own, back issues, hip issues, and things like that...and this is when machines came to the forefront and so that has been a huge help for me so that I could still feel like I was doing the best job that I could for my patients but also not putting myself in danger of injuring myself..."

"I definitely think the lift equipment has helped, but still a lot of us are sore and stiff most of the time."

“...the [manual standing and raising aid], I think that's a huge saver of injuries personally."

"...we talk amongst ourselves...oh that was a really heavy lift...we should have utilized the [manual standing and raising aid] or something to help us out. So, yes, I do see without the equipment, it's likely for injury amongst ourselves."

\section{Self-Management Measures for Work-Related Pain}

Self-management of pain at work was a robust area of discussion for this study group. Two primary methods of pain management arose including co-workers treating each other and the use of Over-the-Counter (OTC) medications such as ibuprofen to manage the pain.

Regarding treating each other, participants were quick to state that one of their co-workers who used to work in an outpatient setting would perform hands-on, manual therapy treatments on those in pain as a way to manage their symptoms. This manual therapy approach is common among Physical Therapists working in outpatient-type settings.

"We usually go see one of us...we treat each other."

"We have a few physical therapists who used to work in outpatient and they do a lot of manipulations and readjusting of all of us." 
"I mean we have a couple of PTs that work with us and they're just adjusting us all the time...I mean they're chronically working to adjust us after work..."

"We have a couple of outpatient therapists that came over not too long ago and we put them to good work in our department straightening out our spine and pelvises..."

"Whenever there was a heavier assist, I can almost tell immediately and have one of our PTs here put me back in alignment."

As mentioned above, the second way that participants self-managed their pain was through the use of OTC medications. Some participants described having a bottle of ibuprofen within the rehab department that they shared when needed. However, other participants denied taking OTC medications.

"We take some advil and move on with life."

"We have a community bottle [of ibuprofen]."

When asked how often they take OTC meds for pain at work, "For me, maybe once a week."...other said, "Yeah I don't take anything really ever."

The final aspect of self-management of work-related pain discussed by participants was changing or self-limiting job tasks or having co-workers help short-term. This was not something recommended by managers but rather self-imposed by participants in order to keep working.

"Yeah, I mean sometimes for me, it's as simple as switching sides or switching a dominant arm."

"You tell your teammates and they'll do more."

"I think you limit yourself, probably, in the next days, while you are still injured."

\section{Discussion}

The purpose of this study was to provide an in-depth description of the work performed by rehabilitation professionals in acute care environments including the perceived risk of workrelated injuries and the role of SPHM equipment in promoting safe work practices. Broadly, this study further supports previous research in which patient mobility is described as the primary role of rehabilitation professionals. 5,6,32 Patient mobility was described by participants in this study as anything that involved purposeful and skilled movement of the patient from repositioning in bed, to sitting up out of bed (supine to sit transfer), to transferring from sit to stand, and ambulating.

Central to these patient mobility tasks was the idea of a therapeutic value to the mobility. This was described by participants as prioritizing patient involvement in the mobility task. The central nature of patient mobility combined with the importance of a therapeutic value to the 
patient are unique to the rehabilitation team. Participants in this study contrasted their role with that of nurses and perceived the nursing role to be more oriented to the end goal of getting patients from one point to another safely rather than working to involve them in the process. An in-depth exploration describing the fundamentals of nursing care created a varied and comprehensive list of tasks seen as central to nursing care. Among the multitude of tasks listed, components of nursing care included providing a safe environment, communication, breathing support, eating and drinking support, elimination, cleansing and dressing, rest, controlling body temperature, mobility, work and play, and end of life care.$^{52}$ In contrast to our study among rehabilitation professionals where patient mobility is described as the central work task, the scope of practice among nurses is much more broad and varied. It is, therefore, no surprise that the focus and needs when performing patient mobility are also varied among these two work groups.

Despite this central role of patient mobility and its associated injury risk, the participants in this study denied experiencing injuries at work. When asked about their work-related injury experiences, they reported frequently working in pain but did not believe these to be workrelated injuries. This is supported by hospital injury data in which zero members of the rehabilitation team reported injuries in the five years since SPHM program implementation. Additionally, they perceived the risk and prevalence of injury as lower among rehab professionals than in other comparable work groups, notably nurses. However, recent research in this area is beginning to suggest otherwise. A recent study comparing nurses and rehabilitation professionals in an acute care setting found that the 12-month prevalence of low back pain among PTs was very high (73.1\%), significantly higher than reported by the nurses (43.9\%), even after adjustment for age, BMI, and morbidity. ${ }^{5}$ Therefore, rehab professionals experience similar, if not greater, incidence of work-related injuries during patient mobility tasks as nurses.

Our findings suggest that the choice of wording when asking about work-related injuries among this work group may be important. When asked, all participants in our study denied injuries at work but were quick to state that they experienced pain due to work-related tasks during or after work-related tasks. Using the word pain versus injury seems to be more acceptable and may result in more accurate reports of work experiences. A nationwide survey by Campo et al, found that when using a case definition of pain rated at 4/10 (on a 0 to 10 scale) lasting more than a week or present at least once a month, nearly $60 \%$ of Physical Therapists reported work-related pain during their career and $20 \%$ reported a new incidence of workrelated pain within a one year follow-up period. ${ }^{4}$ This case definition did not include the word "injury". This was also seen in similar work using the same case definition in which $18 \%$ of Occupational Therapists and $16 \%$ of Physical Therapists reported a one year incidence of workrelated musculoskeletal symptoms. ${ }^{6}$

Despite rarely acknowledging work-related injuries, rehabilitation professionals in our study reported that the use of lift equipment did seem to decrease their pain and risk of injury. This was especially true with the manual sit to stand lift that they used most commonly. This perception of the role of lift equipment in decreasing injury risk is consistent with a similar study among Physical and Occupational Therapists in which the results of focus groups indicated an acknowledgement of the role of the lift equipment in decreasing injury risk. ${ }^{27} \mathrm{~A}$ recent narrative 
review of the literature regarding WMSDs among Physical Therapists described "self-protective behaviors" to avoid injuries and included mechanical lifts and slippery sheets as part of these strategies. ${ }^{7}$ However, when directly comparing rehab professionals and nurses, Myers et al found that nurses were much more likely to acknowledge the protective role of lift equipment in decreasing injury risk. ${ }^{35}$ Another study among nurses reported that a higher perception of injury risk was associated with lack of availability of lift equipment. Conversely, they also reported feeling safer when using the lift equipment. ${ }^{53}$

When participants in this study experienced work-related pain, none reported the injury to their employer but instead chose various methods of self-management. These self-management strategies included treatment by co-workers, informal alteration in job tasks, and use of overthe-counter (OTC) medications. This is similar to several previous studies. Campo et al reported Physical Therapists were not likely to report a work-related injury or seek medical treatment for the work-related pain. ${ }^{4}$ The most common method of self-management among this study population was treatment by a co-worker. Participants in our study often sought the care of coworkers who performed manual therapy treatments to the spine in order to help them manage work-related pain. A recent systematic review regarding work-related injuries among Physical Therapists found that over $61 \%$ of the PTs indicated that they had treated themselves or had sought treatment from a colleague for a WMSD. ${ }^{7}$ A similar qualitative study with Physical Therapists who had been injured at work also reported seeking treatment from colleagues or other Physical Therapists. ${ }^{34}$

Informal alteration of work tasks was mentioned in this study and has also been reported in several previous studies among rehabilitation professionals. In a survey of Physical and Occupational Therapists in the mid-west, almost all participants continued to work even with a work-related injury and many of these reported altering their work habits due to the injury. ${ }^{6} \mathrm{~A}$ survey of Physical Therapists and Assistants also reported that about $25 \%$ of therapists changed their work position in response to a work-related injury and about $40 \%$ increased their use or reliance on other members of the healthcare team. ${ }^{54}$ Several participants in our study also reported using OTC medications (primarily ibuprofen) to manage their symptoms. This avenue of self-management was either not reported in prior work or was denied by rehabilitation professionals who participated in previous research. ${ }^{34}$

Self-management of work-related injuries is not as widely reported in the nursing population. A study by Alperpovitch-Najenson et al, found that nurses were much more likely to miss work than Physical Therapists and, therefore, did not need to self-manage or self-alter job tasks as frequently. ${ }^{5}$ This depicts another difference in work-related injury and pain experiences between these different work groups.

As with any research study, there are both strengths and limitations to this project. The primary limitation of this study is the fact that this is a single-site study. Since qualitative case studies are not meant to be generalizable, comparisons must be made carefully and within the context of this setting and comparable settings in mind. In this case, it is worth noting that this setting was a teaching hospital in a small urban community and that comparisons outside this kind of setting are particularly problematic. However, the SPHM program implemented is structured similarly to other SPHM programs nationwide and these findings may, therefore, be 
transferrable. In addition, our findings are similar to those demonstrated in other studies among rehabilitation professionals. More research at different study sites with varied methodology would help to validate these findings.

However, several study strengths also exist. These include the presence of a strong, comprehensive SPHM program at this facility, the willingness of the hospital employees to collaborate with this project, and the qualitative approach. The presence of an existing but relatively new SPHM program makes it an ideal time to study program effectiveness. This program meets all recommended criteria for an SPHM cited in several sourses. ${ }^{17,19,25,36}$ This includes high levels of equipment purchasing and strong support at the administrative and personnel levels. Additionally, as previously mentioned, nearly $50 \%$ of the targeted population volunteered to participate in this study. Themes emerged repeatedly among the multiple focus groups indicating that saturation had been reached regarding the themes described in this study. The study purpose of discovering the specific details behind equipment use and needs as lends to the appropriateness of this qualitative collective case study to evaluate the rehabilitation professionals' perspectives of this SPHM program.

Additionally, it should be noted that several lessons were learned along the way that were magnified by the timing of data collection during the COVID-19 pandemic. The first three focus groups were held in person several months prior to the existence of COVID-19 (fall 2019). Even with strong recruitment efforts, only the minimum of three participants per focus group was met for each of these three sessions. The last three focus groups were then required to be reorganized for participant safety and were held via Zoom in the spring/summer of 2020. This, in combination with increased incentivization to all participants both past and present, seemed to improve participation. These latter three focus groups had five, eight and three participants respectively. This is most likely due to their already demanding schedules with little time for breaks. A platform such as Zoom that allows participation at a time and place that is convenient for busy healthcare practitioners should be strongly considered for future research.

\section{Conclusion}

Rehabilitation professionals overwhelmingly described patient mobility as the primary aspect of their job. Central to these patient mobility tasks was the idea of a therapeutic value to the mobility. However, despite the fact that these patient mobility tasks have been shown to increase the risk of work-related injuries, participants in this study did not perceive work-related pain as injuries and did not report injuries to their employer. Therefore, in order to maximize the safety of this distinct work group, several recommendations for SPHM trainings and future research should be considered. SPHM trainings should include examples of therapeutic use of lift equipment for rehabilitation professionals in order to maximize their participation in SPHM programs. Additionally, when measuring the effect of these SPHM programs on this work group, hospital injury records should not be used but rather information gained directly from the workers. Researchers should be careful to include the terms work-related pain instead of workrelated injury when asking about the adverse effects of patient mobility. 


\section{Paper 3}

Title: An In-Depth Exploration of Administration Policies and Procedures Guiding Successful Safe Patient Handling and Mobility (SPHM) Program Implementation Among Rehabilitation Professionals

Abstract: (355 words)

Introduction - Safe Patient Handling and Mobility (SPHM) guiding documents state that administrative policies and procedures are a required part of successful and comprehensive programs. However, in research studies evaluating SPHM program success, the administrative and organizational process is either not considered or only mentioned as a vague program requirement. This study provides an in-depth qualitative exploration of the details of SPHM program organization and administration that can influence program success among rehabilitation professionals working in a hospital setting.

Methods - A qualitative case study approach was used to investigate the role of administrative factors on the effectiveness of an ongoing, comprehensive SPHM program as it was applied to rehabilitation professionals. Three semi-structured interviews were conducted with key informants including one manager and two administrative staff involved in overseeing the SPHM program and the Rehabilitation Department. In addition, administrative policies related to the SPHM program were reviewed. Six focus groups were also conducted with 25 members of the rehabilitation team (11 Physical Therapists, 11 Occupational Therapists and 3 Physical Therapist Assistants). Interviews and focus groups were recorded and de-identified transcripts were analyzed for emergent themes.

Results - Several aspects of work organization were identified as contributing to the success of the SPHM program. A dedicated SPHM training department and staff that provide interactive training to all involved in the SPHM program was perceived to be more beneficial than "on the floor" training. SPHM administrative practices such as lift equipment audits and lift screening tools were identified as important parts of SPHM program success but careful consideration as to their execution and involvement of staff should be considered. Barriers to success including sling availability, time, space/room design, and cost were noted. Finally, the potential for workplace cultural dynamics to alter the success of these programs came through as well.

Conclusion - Hospital administration should carefully consider the administrative policies and procedures created as part of comprehensive SPHM programs. Specifically, a lift equipment audit process incorporated into the SPHM program could help improve lift equipment adoption and, therefore, improve worker safety. Additionally, a budgeting program housed outside of each unit or department to purchase SPHM equipment needs is recommended for program longevity. 


\section{Introduction}

Though less studied than other healthcare professions, several recent studies have quantified work-related injuries among rehabilitation professionals (Physical and Occupational Therapists and Assistants). ${ }^{4,6}$ In a prospective cohort study among Physical Therapists nationally, Campo et al found a one year incident rate of new musculoskeletal disorders of $20.7 \%$ and a prevalence of overall musculoskeletal disorders of $57.5 \%{ }^{4} \mathrm{~A}$ similar study by Darragh et al found an incident rate of 17/100 full time equivalents (FTEs) in a regional survey of 1,189 physical and occupational therapists. ${ }^{6}$ The most recent Bureau of Labor Statistics (BLS) Survey of Occupational Injury and IIIness (SOII) data in 2018 included 610 reported injuries among physical therapists and 630 injuries among occupational therapists for an injury rate of $32 / 100,000$ FTE for physical therapists and 77.2/100,000 FTE for occupational therapists. ${ }^{1,12}$

Many of these injuries among healthcare workers are related to patient mobilization. ${ }^{3-5}$ This is especially true for nurses, physical therapists and occupational therapists who regularly perform patient mobility tasks. ${ }^{4,6-11}$ These tasks include repetitive lifting (moving and repositioning) as well as the inadvertent use of sustained or repetitive awkward posturing during these activities. This, combined with the increasing size and dependency levels of patients, aging of both employees and patients, and decreased staffing levels within inpatient facilities, all place healthcare workers at a high risk of musculoskeletal injuries. ${ }^{4,6,8,9}$

To address this problem, several hospitals have implemented Safe Patient Handling and Mobility (SPHM) Programs. These programs are comprehensive in nature and include interventions at each of the three levels of engineering, administrative and personal/behavioral controls. ${ }^{13-18}$ This includes the purchasing and ongoing care of the appropriate types and amounts of lift equipment, adoption of policies regarding the use of these devices, and support for employees using the devices via the establishment of ongoing training and program leaders. ${ }^{17}$

The use and effects of this lift equipment has been readily studied and found to decrease the risk of work-related injuries among these work groups. ${ }^{20,21,24,25,36}$ However, the organization, structural and administrative components of these SPHM programs are much less reported. In his exploration of patient safety in healthcare, Sidney Dekker discussed the idea that healthcare is one of the only industries with a central focus on safety to resist the ideas of systems approaches and theories. He states, "healthcare has a strong preoccupation with individuality." 37 He goes on to discuss how this focus solely on the individual interactions among healthcare practitioners and patients misses the numerous other factors that result in poor employee and patient outcomes. The Human Factors Approach to Ergonomics discussed in the book proposes that we take a broader look at the circumstances of the healthcare system and ask, "How could we change or redesign the system so that it would be difficult or even fallible for humans to make mistakes?" This is especially applicable to comprehensive, system-wide programs such as Safe Patient Handling programs. Without a broader explanation of the system in which these programs are employed, their successes and failures are not fully explained and, more importantly, their ability to be maximally effective and sustainable are missed. 
In a comprehensive study looking at SPHM program implementation at a large teaching hospital in North Carolina, Schoenfisch et al state that we must look at factors beyond just quantitative employee injury counts and rates as measures of successes for these programs. ${ }^{38}$ Through an in-depth qualitative exploration of focus groups and interviews, they found that factors such as unit and room layouts, storage spaces and processes, and other organizational factors might affect the successes or potential failures of these programs. They suggest it is crucial for further research to include the contextual information of the organization in which the program is being implemented in order to maximize program understanding and effectiveness.

Additionally, many of the current systematic reviews on workplace injury reduction programs are inconclusive or deemed not effective as most studies focus primarily on the work and workers and do not explore the effect of the administrative practices and policies. ${ }^{39-41}$ Several recent studies have demonstrated the effectiveness of including and exploring organizational policies and practices on various aspects of workplace injuries. Tveito et al, explored the impact of organizational policies and procedures in the hospital setting and found that injury rates decreased in units where nurses reported positive or higher perceptions of the organizational policies and procedures. ${ }^{42}$. Therefore, the purpose of this qualitative research project was to explore the specific details of healthcare administration that help to determine the effectiveness of these SPHM programs among rehabilitation professionals as they participate in SPHM programs.

\section{$\underline{\text { Methods }}$}

\section{Research Design}

A qualitative case study design was employed given that the primary study purpose was to provide an in-depth description and analysis of a bounded system. ${ }^{43,44}$ In this case, the bounded system was an SPHM program. This approach allows researchers to study complex phenomena in their natural setting and seeks to provide a rich, holistic description of the issues from the perspective of participants. ${ }^{43,44}$ The participants are selected based on the study purpose and what they can reveal about the system or topic of interest.

\section{Study Site and Program Description}

The study site was a nearly 700 bed academic medical center with a hospital-wide SPHM program established in 2015. The SPHM program included a multilevel implementation strategy that consists of mechanical lift equipment, policy changes for patient lifting, and establishment of program trainings and lift team meetings. The planning and establishment of this program was led by the hospitals safety department. The equipment purchased included equipment that could assist with the performance of supine to sit transfers, mechanical and manual standing and raising aids, lateral transfers and repositioning devices, floor lifts, and ceiling lifts. ${ }^{45}$

\section{Investigator Perspectives}

The team of investigators included two Physical Therapists (one with an academic doctorate in Occupational Health with extensive research experience within this field), three 
occupational health research scientists (one with extensive research experience within this field and within similar SPHM programs), and an occupational medicine physician. This diversity of backgrounds helped enrich the perspectives on the study findings. The research team often discussed and recognized their own contributions and biases during the research process.

\section{Sampling Strategy and Data Collection}

Due to the qualitative methodology of this study, purposeful sampling methods were employed. ${ }^{44}$ This approach intentionally samples a group of people that can best inform the researcher about the research problem under examination. ${ }^{44}$ Key informants (managers and administrative personnel who were involved in the planning or execution of the SPHM program) were recruited via in person meetings and word of mouth. Focus group participants (rehabilitation professionals involved in patient mobility tasks throughout a majority of their day) were recruited via emails through study site gatekeepers using a recruitment flyer. Interested participants for both groups earned a $\$ 20$ gift card for their participation that was later increased to $\$ 140$ in gift cards to increase participation broadly within the study. This study was approved by the West Virginia University Institutional Review Board and consent was verbally obtained via a cover letter before the start of each interview and focus group.

Interviews were chosen as the primary method for data collection with managers and administrators due to their dissimilar job titles and the potential for participant discussion that may not have elucidated study themes. ${ }^{55}$ These interviews were conducted in-person with two hospital administrators and one manager who had administrative roles in the development or ongoing execution of the SPHM program. Each interview lasted between 30 and 60 minutes and was guided by semi-structured guides. The guide was used to ensure discussion of work tasks and procedures, work-related injuries, SPHM program roles and experiences, and organizational effects on the SPHM program. Additionally, several supporting documents were collected for analysis including the Safe Patient Handling Policy Manual and two SPHM training manuals.

Focus groups were chosen as the primary method for data collection with rehabilitation professionals as this allowed for participant discussion and interactions about this SPHM program. Focus groups are beneficial for discussion of topics that could be discussed comfortably in everyday life. ${ }^{43}$ Focus groups consisted of various combinations of rehabilitation professionals (Physical and Occupational Therapists and Assistants) and were scheduled and structured based on participant availability. Each focus group lasted between 60 and 90 minutes and were guided by semi-structured guides. The guide was used to ensure discussion of work tasks and procedures, SPHM program experiences, equipment use, equipment needs, barriers to equipment use, and organizational effects on SPHM programs. Focus groups were initially held in person, however, the COVID-19 pandemic forced the remaining three focus groups to occur via Zoom.

In-person and Zoom focus groups and interviews were recorded using digital voice recorders or the built-in Zoom recording feature. Participants used alias names during the focus group process to ensure anonymity. The primary investigator also took field notes during each 
focus group and interview. The data was transcribed using the human transcription option through Rev.com. ${ }^{46}$

\section{Data Analysis Procedures}

Broadly, a content or thematic coding analysis structure was used to analyze the results of both the interviews and focus groups. The primary and secondary study investigator independently analyzed the transcribed data using first and second cycle coding procedures. ${ }^{48}$ First cycle coding procedures chosen included attribute coding, descriptive coding (topic based) and in vivo coding (terms used by participants themselves) initially using a sentence by sentence initial coding process and then holistic paragraph by paragraph coding to develop open code categories. ${ }^{48}$ First cycle coding was performed by hand and then uploaded to Microsoft Office Word for organization and secondary coding and analysis. The documents obtained were analyzed similarly for attribute and descriptive codes and then added to the Microsoft Office Word document for organization.

\section{$\underline{\text { Results }}$}

\section{Study Participants}

Three hospital administrators participated in three separate interviews conducted over a two-month period. These hospital administrators included two members of the Safety Department that are intimately involved with the SPHM program policies, administration and training. The third interviewee is responsible for management of the Physical and Occupational Therapists within the Rehabilitation Department. Twenty-five rehabilitation professionals (11 Physical Therapists 11 Occupational Therapists, and 3 Physical Therapist Assistants) participated six focus groups over a nine-month period.

\section{Themes}

Four primary themes emerged from the interview and focus groups. These included the components of training in program success, administrative policies and practices that affect program success, common program barriers, and the role of culture in work safety programs. Some of these emerging themes were consistent between administration and rehabilitation professionals (common program barriers, and the supportive role of a successful training program) while others were only mentioned by managers and administrative personnel (the role of the lift screening tool, the data gathered during the injury reporting process, and administrative equipment audits).

\section{The Components of Training in SPHM Program Success}

A large portion of the discussion in each of the interviews centered around the various aspects of SPHM training program. During the interviews, the various components of the training program, including its evolution, as well as administrative perspective on its success were discussed. Support for this was found in the SPHM Policy document. Focus group 
participants primarily cited the positive perceptions of a rehabilitation professional conducting the training.

\section{$\underline{\text { Administrative Staff Interview Data }}$}

"...we make sure that we explain the equipment, we demonstrate how to use it, and then all staff have to get hands on to show they understand how to use the equipment."

"So, a few years ago we were able to hire two full-time trainers and their background was a PTA and that's all they do. They train new hires. Anybody who lays hands on patients for mobility whatsoever gets trained and they give them different kinds of training."

"I think it's good that the hospital decided to have a dedicated class for this, because previously we bought the equipment... we had the consultant doing the training...she would just kind of train people in units as they were available...but we found that turnover in general was an issue."

"...we make them fill out a post survey and we always get good reviews...occasionally we'll get somebody that says it's either too long of a class...and then sometimes you'll get people that say it's too quick of a class...but overall people...they're pretty responsive to it."

\section{Department Manager Interview Data}

"Hands down, the thing that is going well is that we have two PTA's that do the training. Hands down, that's the best thing."

\section{Policy Document Data}

"Proper training on the use of the equipment is essential to maximize benefits for employees and patients...New employees will receive training for Safe Patient Mobility prior to the initial use of equipment and within 30 days of start date for positions where patient lifting is required. No employee shall use Safe Patient handling equipment prior to completion of the training. Training will include live training conducted by the Safe Patient Mobility Specialists."

\section{Focus Group Data}

"I think it's good that we have therapists teaching nursing staff about equipment use, because they kind of understand more of the mechanics of it from like a patient mobility perspective."

"It's really awesome that they have that [PTA] background and I've actually attended their class.... So that's nice. Just that therapy aspect helps a lot I think." 
"And the class is taught by physical therapy assistants that used to work here daily and see patients daily. So I mean, they know what we go through on our end. So, it is a good course.

\section{Administrative Policies and Practices that Affect SPHM Program Success}

Equipment audits, program financing, the use of a lift screening tool, and injury investigations were discussed in relation to the SPHM program. Equipment audits performed by the safety department were discussed in terms of the process itself, the components evaluated, and the distribution of the results. This process was not discussed during the focus groups nor mentioned in the SPHM Policy document.

\section{$\underline{\text { Administrative Staff Interview Data }}$}

"Oh the audits...so we just randomly go onto the floors, we write what unit we're on, and we'll just grab people. We don't ever write names...just if they're a nurse or CA...and then we ask them, 'Do you know where the equipment is located? Do you know if you need slide sheets or slings and they're not in your clean utility, who do you call? Do you know you have a TMC or super user? Do you know there's a website?' And then we write if they're using any equipment... are they using it correctly, are they using the correct equipment?"

"And then on the back of the paper, we just have equipment stocking. So this is where I'll write what piece of equipment they have."

"I can do the usage meter to see how many times they've used that piece of equipment, and then compare it for later."

"We're trying to keep it to a one page summary [of audits] for managers."

Program financing was discussed only during interviews and occurred initially through a large capital request paid for out of the hospitals' capital budget. However, once this initial program investment was completed, additional equipment or program needs or purchases must now be part of each unit's yearly budget. The department manager discussed the difficulty of this regarding timing and balancing the number of equipment requests.

\section{$\underline{\text { Administrative Staff Interview Data }}$}

"Initially our budget, Safety's budget, which is basically the hospital's budget, footed the bill for all of it [SPHM program]...So, now it's up to each unit to purchase the equipment they need."

"So we [safety department] can make recommendations, but all equipment is that unit's budget."

\section{Department Manager Interview Data}


"The hard part though, again, budget and getting staff to come to us at the right time in the budget cycle for equipment...We haven't gotten everything they've asked for, but we've got several."

The LIFT (Living Injury Free Together) Screening Tool was developed as a joint effort between hospital administration, staff and the equipment company (Arjo). Participants felt this was intended more for nursing than rehabilitation professionals to help them determine the best way to mobilize a patient in or out of bed, including whether or not lift equipment is needed and which device is most appropriate to use. A description of this tool and its execution process were found in the SPHM Policy document, however, the employees required to use this tool was not specified.

\section{Administrative Staff Interview Data}

"So they do that upon admission and every 12 hours or with status change of the patient, and it determines...according to the answer given, it cascades down what devices or equipment you would use on the patient."

"So, between [the equipment manufacturers] recommendations and our core team, we developed it."

"It [the LIFT screening tool] is in [our electronic health record system]. The problem is...that it's not a hard stop. You can get past it. It's under 'required' but it's not a hard stop where you can't go any farther until you complete it. So they do work arounds."

\section{Department Manager Interview Data}

"Yes I don't necessarily know that our staff use that [lift screening tool] a great deal to make their decisions, because when lift training came through, they trained nursing significantly different than what they trained rehab services at the time. They trained nursing on how to screen to decide which tool to use to lift a patient for just daily care activities."

\section{Policy Document Data}

"Patient's mobility needs will be assessed upon admission, in conjunction with the fall risk assessment, and with any change in physical status. The lift equipment and assistive devices are listed in the electronic medical record under Activities and Interventions and should be evaluated when charting the patient's current condition. The patient profile screening, LIFT Tool will be used in determining equipment needs."

The injury reporting process surrounding patient mobility and lift equipment use was also discussed. The Safety Department at the hospital determines if the injury was preventable through the use of lift equipment and if an employee is found in violation of the lift equipment 
policy, further SPHM training is required. This was discussed only by administrative personnel and did not arise during other interviews or focus groups.

\section{$\underline{\text { Administrative Staff Interview Data }}$}

"So if somebody gets hurt, there is a form that has to be filled out, and then that form gets sent to employee health, they take a look at it. If it's a patient handling issue, they send it to the safety department, they look at it, they decide, is it a preventable injury or not? Then if it's a preventable injury, then they see how did it occur. Was it because they didn't use equipment, was it they didn't use it right? And then they send it on to me and I get to see the report, I see the injury, and then I say, 'Well this should have been used."'

"...then the Safety Department sends that information back to the management or director of that unit to address it...And then they need to go through another class and sit through it."

\section{Common SPHM Program Barriers}

Program barriers cited throughout the focus groups and interviews were broadly similar and included sling availability, time, space/room design, and cost. The lack of proper sling stocking on the floors combined with the time it takes to get a needed sling from facilities were discussed as barriers to equipment use that requires a sling.

\section{$\underline{\text { Administrative Staff Interview Data }}$}

"One of the things we feel may be the issue is your two other active pieces, they require slings...so you have to get from materials, so if it's not stocked, you have to call materials and wait for them to send it up, which can take you two hours to get depending on how busy they are...[and] they both require batteries, so you have to make sure your batteries are charged."

"Making sure you have the slings stocked, making sure that people...know where to get them."

\section{Focus Group Data}

"The sling stocking is a little hit or miss in certain units because I don't think someone really inventories."

"It's hard to find the slings and stuff that you need for the certain specialty pieces of equipment.

"Its just initially getting the sling can be the biggest barrier. You have to call materials."

"We can never find the right slings that we need. Because there's a different sling for sitting, a different sling for standing, and a different sling for walking, and different sizes 
for patients. And we don't have access to the majority of these slings anymore. They went to the laundry and never came back or they get lost up on the floor and are never replaced."

Time, spatial design and overall equipment cost were also cited as barriers to optimal program success. Time was discussed by administrators as something that was cited as a barrier by employees but that they perceived as not much of an issue. Administrative personnel also discussed the barriers of purchasing and installing ceiling mounted lifts. Rehabilitation professionals also discussed spatial barriers to equipment use but their spatial concerns were different than those of administration.

\section{$\underline{\text { Administrative Staff Interview Data }}$}

"Nurses are using their draw sheet and just going. We have been told that they feel its extra time to use the slide sheets and they say, 'We don't have the time."'

"...and we have been told they won't get any because there's not enough room between the ceiling and what's above them to put reinforced steel beams to hold it."

"And I don't know what their budget is but I guarantee it's tight already. And for you to be like, 'I've got to come up with $\$ 10,000$ [for a ceiling mounted lift] is not an easy feat."

\section{Department Manager Interview Data}

"I think that especially for our staff that bring patients to the gym, I think they're more likely to use that equipment because they have that support to get everything set up, they know where all the slings are. It doesn't creep into making their prep time for patient care longer."

"I think just continuing to find ways to get these devices into your treatment plan, because even though it may not save time, I don't think it particularly increases the amount of time you spend with a patient on that individual session."

\section{Focus Group Data}

"The chairs in the patient's room aren't always compatible. So like the legs on the [manual standing and raising aid] are too high and they don't fit underneath of the chair, so you can't get the patient directly over the seat of the chair. There's kind of a gap."

"...sometimes like the layout and space in the room can make it difficult to use some of the equipment, especially the [floor lift]."

"sometimes the setup of the rooms... and they are oddly shaped...so there's certain rooms that are horrible for [equipment and mobility]."

$\underline{\text { Role of Culture in Workplace Safety Programs }}$ 
The term culture or workplace culture were not terms overtly mentioned by participants but the themes that belie culture were discussed. These included workplace conflicts regarding the way things are versus should be done. The role of employee age and experience with these "shop floor norms" were also discussed.

\section{Administrative Staff Interview Data}

“...new hires, they'll be like, 'This is awesome. This is great.'...then once these new people get on the floors and they're really excited about it, then our seasoned staff are like, 'We don't do that here."'

'You know you've got this dinosaur thinking and these nurses, even though they've had the training at work, we never got hurt before... and they don't get that they just have been lucky that they could have career ending injuries because you didn't have time to put the slide sheets out."

"And during my investigations, l've found that they're not following policy, they're not using the slide sheets and these are people who know better. So our VP of nursing has taken this task on of looking into these and never did we want discipline to be the encourager for compliance but it may have to come to that."

"They're just not following policy."

\section{Department Manager Interview Data}

"I don't know about the newer therapist, but I think I would even consider it with some of the older therapists, that we do take pride in scoffing at that 35 pounds [NIOSH lifting limit in healthcare]."

\section{Discussion}

In SPHM guiding documents it is often noted that administrative policies and procedures are a required part of successful and comprehensive programs. ${ }^{13,14,18}$ However, in research studies evaluating SPHM program success, the administrative and organizational process is either not considered or only broadly mentioned as a program requirement. This study provides an in-depth description of the details of SPHM program organization and administration that can influence program success including details of a successful training program, various aspects of policies and procedures that either enhance or inhibit program success, common program barriers, and the role of culture in the execution of these programs.

The specifics of a successful SPHM training program were discussed in both interviews with administration and management as well as in focus groups with employees. The training program at this specific facility has evolved over time and study participants were supportive of its current state and role within this SPHM program. The components that were viewed as successful deserve consideration within other SPHM programs. These include having a 
dedicated training department and program outside of those otherwise involved in the program with training required for all employees that includes equipment explanations, demonstrations and hands-on practice. The other aspect of this training program that was consistently mentioned as a program facilitator is that the primary trainer(s) are rehabilitation professionals who were perceived as having a strong understanding of the needs and requirements of successful lift equipment usage. In a recent two part series, several of these components of SPHM training were recommended as best practice including requiring these trainings as part of new staff orientation and including hands-on equipment practice and problem solving opportunities within the training programs. ${ }^{56,57}$

The SPHM program policies that guide program implementation and execution must also be considered when determining program success. For this specific program, the administrative items that came to light were the use of program audits to determine lift equipment use, the budgeting specifics, the components of the injury reporting process, and the use of a lift screening tool. Lift equipment audits are performed periodically by the hospitals safety department to look at equipment usage as well as various reasons for and limitations to usage. Safety administrative personnel perform these audits that include discussions with employees (typically nurses) and evaluation of equipment storage and stocking. The intent with these is to help employees overcome barriers to equipment usage and provide strategies for improvement in the future. However, with both the injury reporting and injury audit process the reference for employees to follow the policies in place was often mentioned. A systematic review on workplace interventions found two studies of medium and high quality that demonstrated a positive effect on worker behaviors when combining training and audits together in the workplace.$^{58}$ More specifically, SPHM program audits are recommended as a component of ongoing SPHM programs in order to observe patient mobility tasks, gather staff feedback, and provide coaching regarding equipment usage. ${ }^{59}$

The role and location of SPHM program budgeting was also discussed and may warrant careful consideration when considering the longevity of these worker safety programs. For this particular study, administration and managers noted that a capital, hospital-wide budget responsible for initial program funding and purchases. Although not explicitly stated by participants, this seemed to allow for wide-scale purchasing of equipment as needed and recommended for successful program initiation. However, ongoing budget requests and lift equipment purchases are now up to each department's budget. This brings up much more variability in the ability of different department to purchase lift equipment to meet the ongoing needs of employees.

Within this particular facility, injury reporting is a multi-step process in which employee health determines whether or not the reported injury is "preventable" based upon reports gathered from both the employee and unit managers. If the work-related injury occurred during patient handling, administration within the hospital's safety department assists with this process and helps to determine if lift equipment was and/or should have been used. If it is determined that lift equipment was not used or not used properly, the employee is required to undergo further training and education to try to help ensure that similar patterns of practice do not continue. A mixed-methods study by Siddhartan, et al, regarding underreporting in Veterans Administration healthcare employees, found that time was often a barrier to injury reporting 
among these employees. ${ }^{60}$ Time includes both the reporting process as well as any remediation that is deemed necessary to occur. Within this particular facility, time required to repeat these trainings may be beneficial for these employees but could be a significant barrier to reporting injuries in workers with already busy work days and patient caseloads.

In order to help employees determine when lift equipment usage is appropriate, a lift equipment screening tool was created within this facility. This was a joint effort between hospital administration and the lift equipment company. ${ }^{45}$ The Living Injury Free Together (LIFT) Screening Tool was created more for nursing employees than any other employee subgroup and helps them determine the best way to mobilize a patient in or out of bed, including whether or not lift equipment is needed and which device is most appropriate to use. Per the hospital's SPHM policy manual, "Patient's mobility needs will be assessed upon admission, in conjunction with the fall risk assessment, and with any change in physical status. The lift equipment and assistive devices are listed in the electronic medical record under Activities and Interventions and should be evaluated when charting the patient's current condition. The patient profile screening, LIFT Tool will be used in determining equipment needs." However, it was reported by administration that even though this tool is required in the Electronic Health Record (EHR), employees have found ways to circumvent performing this tool as required.

Although well-intended, administrative policies such as these have been found to be out of touch with the way work is done on the floor and adds to an already heavy administrative burden for employees whose primary job is to provide patient care. An ethnography of nursing assistants in skilled nursing facilities describes, in depth, the shortcuts employees are often forced to create in order to complete all required tasks in a job shift, even in facilities that are considered adequately staffed. ${ }^{61}$ This study specifically describes the difficulties with using lift equipment as prescribed by administration and administrative policies. This includes time required to use the equipment and assistance needed to do so correctly, both of which are difficult to come by in the fast-paced world of healthcare.

Similar SPHM program barriers were discussed in both interviews with administration and focus groups with employees. They included concerns with sling availability, time and structural/spatial limitations. The specific concerns with slings were the lack of availability when needed on units that then required a call to the Materials Department for a sling that could take hours to days for the sling to arrive. This was noted in both interviews and focus groups. Time was cited as a barrier to use only in the interviews with administration. They reported that their employees often cited time as a barrier to use but their perspective was that it did not seem to increase overall patient treatment time. Structural and spatial barriers were different between administration and employees with administration concerned with the inability to retrofit ceiling lifts into some rooms. For employees, the primary spatial barrier was inability to fit equipment into rooms and around existing items in room (such as bedside chairs). These types of SPHM program barriers have been reported in various other studies. ${ }^{27-29,50,51}$

"Workplace culture" or "safety culture" are terms commonly used in today's work place and are often used to describe explicit or tangible representations of culture, such as decreased injury rates or costs. However, sociologists and anthropologists who study culture extensively explain that culture goes beyond these tangible items. They remind us that culture is our way of 
making sense of the world and it, therefore, provides a framework for understanding various aspects of the workplace. ${ }^{62}$ Culture will therefore affect workers decisions on how to complete work tasks and, therefore, may inadvertently affect injury risk. ${ }^{35}$ Sociological research studies regarding work have shown that employees will create "shop floor" norms in order to be successful at work. ${ }^{61}$

In this particular study, the use of the term "culture" did not arise when discussing patient mobility and SPHM programs. However, inferences to "shop floor" norms did arise during these administrative interviews. These included workplace conflicts regarding the way things should be done versus the way they are and how these conflicts may arise based on employee age and experience. The conflict that arises when new employees complete SPHM lift equipment training with enthusiasm and are then met with resistance on the work floor by more experienced employees was discussed. It was also mentioned by administration that the use of "the old way" of doing things (without using the lift equipment) seemed to be pervasive among experienced nurses who had not experienced a work-related injury. This type of conflict and resistance to change is not new in any organization, healthcare included. Therefore, methods to integrate new procedures into existing processes should be considered. This could include ongoing lift equipment training programs repeated periodically (rather than just at hire) to integrate staff and processes. This was actually mentioned by administrators as an SPHM program goal within this facility.

\section{Limitations}

One of the primary limitations of this study is the fact that this is a single-site study. Since qualitative case studies are not meant to be generalizable, comparisons must be made carefully and within the context of this setting and comparable settings in mind. In this case, it is worth noting that this setting was a teaching hospital in a small urban community and that comparisons outside this kind of setting are particularly problematic. However, the SPHM program implemented is structured similarly to other SPHM programs nationwide and these findings may, therefore, be transferrable. In addition, our findings are similar to those demonstrated in other studies among rehabilitation professionals. More research at different study sites with varied methodology would help to validate these findings.

Another potential limitation could be the few number of interviews conducted with administration and management. However, a qualitative case study does not require a large number of participants in order to demonstrate strong methodology. Rather, it requires identification of key informants who can provide information regarding the bounded system being studies. In the case of this study, the administration and management interviewed were identified as such and no further key informants were identified, even when asked at the end of each interview. Additionally, this perspective and study are strengthened by the perspective of the employees who participated in focus groups and were asked about organizational and structural factors affecting SPHM participation.

In conclusion, hospital administration should carefully consider the administrative policies and procedures created as part of comprehensive SPHM programs. Specifically, a lift equipment audit process incorporated into the SPHM program could help improve lift equipment 
adoption and, therefore, improve worker safety. Additionally, a budgeting program housed outside of each unit or department to purchase SPHM equipment needs is recommended for program longevity. 


\section{$\underline{\text { V. Summary }}$}

The results of this study centered around the experience and needs of rehabilitation professionals working in hospital settings with SPHM programs. More specifically, the qualitative collective case study approach provided rich, in-depth descriptions of the needs and uses of lift equipment among rehabilitation professionals, the central job tasks that differentiate this distinct work group from others, their work-related injury experiences, and the organizational and administrative factors affecting SPHM program participation among rehabilitation professionals. These findings should be considered by hospital administrators and equipment manufacturers in order to maximize the work-related safety and experience of rehabilitation professionals.

\section{A. Paper 1 Discussion Summary}

Paper 1 presents the primary study findings regarding attitudes and perceptions of SPHM programs among rehabilitation professionals. The results of this first paper demonstrate broadly supportive attitudes towards SPHM programs among rehabilitation professionals which is consistent with several recent research studies. ${ }^{27,29}$ Clinical decision-making pertaining to the use of lift equipment was also explored and participants discussed this in terms of three main factors - patient characteristics, transfer type, and work safety. Participants in this study preferred to use the lift equipment with patients who demonstrated generalized weakness and deconditioning and for those with cognitive impairments, fear or anxiety. Research studies in this area among rehabilitation professionals is quite variable with use of the lift equipment with being primarily with bariatric patients and those with lower level functioning. ${ }^{27,28}$ In contrast, the nursing literature reported nurses do not use the equipment with patients with increased fear and anxiety nor those with medical lines and tubes. ${ }^{50}$ The variability in the use of lift equipment between facilities and professions warrants further, large-scale studies on how healthcare workers are using the equipment in order to maximize their work safety within these SPHM programs.

Despite the fact that participants were supportive of SPHM programs, they routinely defaulted to manual transfer techniques. Participants indicated that they chose to use the equipment only under certain circumstances including when a manual transfer failed or felt unsafe, during follow up treatments more than initial evaluations, and when they did not have an extra set of trained hands for assistance. Additionally, some participants reported that decreasing the chance of injury has begun to factor into their decision to use the lift equipment. The realization of risk of injury with patient mobility overall among therapists has been discovered in previous studies but has only been more recently discussed as a reason for lift equipment usage. ${ }^{6,28}$ Similar themes are starting to emerge in the nursing literature as well with a recent qualitative study finding concern for personal health as a motivator for lift equipment usage.$^{51}$ Although risk of injury was certainly mentioned by a few participants as a reason for lift equipment usage, the reasoning among these participants was overwhelmingly due to patient characteristics as opposed to their own safety. Hospital administration and equipment manufacturers must start to consider the possible reasoning behind this lack of use, which is discussed below. 
One of the most robust areas of discussion for participants in this first paper centered around the type of lift equipment used. Unequivocally, the manual standing and raising aid was the piece of equipment used most by rehabilitation professionals within this study. The common reasons behind the popularity of this equipment included ease of use, availability, and ability to be used in a therapeutic manner. The popularity of this piece of equipment among rehabilitation professionals has been demonstrated in other studies as well. ${ }^{27}$ This supports the idea that readily available equipment that does not require battery charge or extra parts (such as a sling) may be ideal for improving adoption and use among rehabilitation professionals. Current studies among nurses are finding different patterns of equipment usage than we observed among our study participants. A recent survey among nurses and nurse assistants found that the dependent floor lift and the mechanical sit-to-stand lift were the most commonly used pieces of equipment among that work group. ${ }^{50}$ However, this was a single site study and further largescale studies looking specifically for type of lift equipment usage and preferences among various healthcare workgroups is recommended.

One area of this first paper that, to our knowledge, has not been previously described includes suggestions for equipment modifications for rehabilitation needs. These came up prominently within each focus group. These recommendations were for the manual standing and raising aid and included a bariatric version, wider hip area, removable unilateral footplate, and a height adjustable knee plate. This information addresses the concern raised by similar studies of rehabilitation professionals that these lift equipment devices were not designed with the idea of promoting patient independence in mind. ${ }^{27}$ As mentioned previously, given that this is the most popular piece of lift equipment used among these study participants as well as in other studies among rehabilitation professionals, lift equipment manufacturers must begin to consider these changes in order to maximize lift equipment use among this occupational group as promoting patient independence is so central to their daily work practices. Additionally, hospital administration should also begin to lobby on their behalf for these changes as this will decrease their work-related injury risk and associated costs.

\section{B. Paper 2 Discussion Summary}

The results of the second paper provided an in-depth description of the work performed by rehabilitation professionals in acute care environments including the perceived risk of workrelated injuries and the role of SPHM equipment in promoting safe work practices. Broadly, this study further supports previous research in which patient mobility is described as the primary role of rehabilitation professionals. 5,6,32 Patient mobility was described by participants in this study as anything that involved purposeful and skilled movement of the patient from repositioning in bed, to sitting up out of bed (supine to sit transfer), to transferring from sit to stand, and ambulating. Central to these patient mobility tasks was the idea of a therapeutic value to the mobility. This was described by participants as prioritizing patient involvement in the mobility task.

The central nature of patient mobility combined with the importance of a therapeutic value to the patient are unique to the rehabilitation team. Participants in this study contrasted their role with that of nurses and perceived the nursing role to be more oriented to the end goal of getting patients from one point to another safely rather than working to involve them in the process. In 
contrast to our study among rehabilitation professionals where patient mobility is described as the central work task, the literature describes the scope of nursing practice as much more broad and varied. ${ }^{52}$ It is, therefore, no surprise that the focus and needs when performing patient mobility are also varied among these two work groups. The results of our first study describe some of the specific needs among rehabilitation professionals with lift equipment design.

Despite this central role of patient mobility and its associated injury risk, the participants in this study denied experiencing injuries at work. When asked about their work-related injury experiences, they reported frequently working in pain but did not consider these to be workrelated injuries. This is supported by hospital injury data in which zero members of the rehabilitation team reported injuries in the five years since SPHM program implementation. Additionally, focus group participants perceived the risk and prevalence of injury as lower among rehabilitation professionals than in other comparable work groups, notably nurses. This perception has been reported in previous research that compared nurses and rehabilitation professionals participating in SPHM programs. ${ }^{35}$

However, recent studies reporting injury-related data are beginning to suggest otherwise. A recent study comparing nurses and rehabilitation professionals in an acute care setting found that the 12-month prevalence of low back pain among physical therapists was very high (73.1\%), significantly higher than reported by the nurses (43.9\%), even after adjustment for age, $\mathrm{BMI}$, and morbidity. ${ }^{5}$ Therefore, rehabilitation professionals experience similar, if not greater, incidence of work-related injuries during patient mobility tasks as nurses, however, they are much less likely to report these injuries and prefer to self-manage. ${ }^{4}$ The self-management strategies used by participants in our study included treatment by co-workers, informal alteration in job tasks, and use of over-the-counter (OTC) medications.

In order to maximize the safety of this distinct work group, several recommendations for SPHM future research should be considered. SPHM trainings should include examples of therapeutic use of lift equipment for rehabilitation professionals in order to maximize their participation in SPHM programs. Additionally, when measuring the effect of these SPHM programs on this work group, hospital injury records should not be used but rather information gained directly from the workers. Researchers should be careful to include the terms workrelated pain instead of work-related injury when asking about the adverse effects of patient mobility.

\section{Paper 3 Discussion Summary}

The results of this third paper present a broad view of the administrative and organizational effects on healthcare interventions that is not always considered when reporting successes or difficulties with various workplace safety programs. In SPHM guiding documents it is often noted that administrative policies and procedures are a required part of successful and comprehensive programs. ${ }^{13,14,18}$ However, in research studies evaluating SPHM program success, the administrative and organizational process is either not considered or only broadly mentioned as a program requirement. The results of this study describe a successful SPHM training program and bring to light some considerations for SPHM administration in order to promote continued program success such as lift screening tools and lift equipment audits. 
The training program at this specific facility has evolved over time and study participants were supportive of its current state and role within this SPHM program. The components viewed as successful included having a dedicated training department outside of those otherwise involved in the program with training required for all employees that includes equipment explanations, demonstrations and hands-on practice. The other aspect of this training program that was consistently mentioned as a program facilitator is that the primary trainers are rehabilitation professionals who were perceived as having a strong understanding of the needs and requirements of successful lift equipment usage. Therefore, based on these study findings, successful SPHM training programs should have a dedicated training program and staff with special consideration given to the work training and history of the trainers.

The SPHM program policies that guide program implementation and execution must also be considered when determining program success. For this specific program, the administrative items that came to light were the use of program audits to determine lift equipment use, the budgeting specifics, the components of the injury reporting process, and the use of a lift screening tool. Lift equipment audits are performed periodically by the hospitals safety department to look at equipment usage as well as various reasons for and limitations to usage. Safety administrative personnel perform these audits that include discussions with employees (typically nurses) and evaluation of equipment storage and stocking. The intent with these is to help employees overcome barriers to equipment usage and provide strategies for improvement in the future. Prior research supports SPHM program audits as a recommended component of ongoing SPHM programs in order to observe patient mobility tasks, gather staff feedback, and provide coaching regarding equipment usage. ${ }^{59}$

The role and location of SPHM program budgeting was also discussed and may warrant careful consideration when considering the longevity of these worker safety programs. For this particular study, administration and managers noted that a capital, hospital-wide budget responsible for initial program funding and purchases. Although not explicitly stated by participants, this seemed to allow for wide-scale purchasing of equipment as needed and recommended for successful program initiation. However, ongoing budget requests and lift equipment purchases are now up to each department's budget. This brings up much more variability in the ability of different departments to purchase lift equipment to meet the ongoing needs of employees. Therefore, it is recommended to keep some portion of overall or unitspecific budgeting dedicated to the ongoing lift equipment program purchasing needs.

Within this particular facility, injury reporting is a multi-step process in which employee health determines whether or not the reported injury is "preventable" based upon reports gathered from both the employee and unit managers. If the work-related injury occurred during patient handling, administration within the hospital's safety department assists with this process and helps to determine if lift equipment was and/or should have been used. If it is determined that lift equipment was not used or not used properly, the employee is required to undergo further training and education to ensure that similar patterns of practice do not continue. Within this particular facility, time required to repeat these trainings may be beneficial for these employees but could be a significant barrier to reporting injuries in workers with already busy work days and patient caseloads. 
In order to help employees determine when lift equipment usage is appropriate, a lift equipment screening tool was created within this facility. This was a joint effort between hospital administration and the lift equipment company. ${ }^{45}$ The Living Injury Free Together (LIFT) Screening Tool was created more for nursing employees than any other employee subgroup and helps them determine the best way to mobilize a patient in or out of bed, including whether or not lift equipment is needed and which device is most appropriate to use. Although wellintended, administrative policies such as these have been found to be out of touch with the way work is done on the floor and adds to an already heavy administrative burden for employees whose primary job is to provide patient care. At this particular facility, it was reported in the administrative interviews that even though this tool is required in the Electronic Health Record $(E H R)$, employees have found ways to circumvent performing this tool as required, most likely due to time limitations during a busy workday.

Therefore, hospital administration should carefully consider the administrative policies and procedures created as part of comprehensive SPHM programs. A dedicated safety and training staff that provide interactive and ongoing training will lead to greater equipment usage and worker safety. SPHM administrative practices such as lift equipment audits and lift screening tools were identified as important parts of SPHM program success but careful consideration as to their execution and involvement of staff should be considered. These administrative practices will allow greater success and worker safety for those involved in patient mobility in hospitals with existing SPHM programs.

\section{Strengths, Limitations and Lessons Learned}

The primary limitation of this study is the fact that this is a single-site study. Since qualitative case studies are not meant to be generalizable, comparisons must be made carefully and within the context of this setting and comparable settings in mind. In this case, it is worth noting that this setting was a teaching hospital in a small urban community and that comparisons outside this kind of setting are particularly problematic. However, the SPHM program implemented is structured similarly to other SPHM programs nationwide and these findings may, therefore, be transferrable. In addition, our findings are similar to those demonstrated in other studies among rehabilitation professionals. More research at different study sites with varied methodology would help to validate these findings.

Another potential limitation could be the few number of interviews conducted with administration and management. However, a qualitative case study does not require a large number of participants in order to demonstrate strong methodology. Rather, it requires identification of key informants who can provide information regarding the bounded system being studies. In the case of this study, the administration and management interviewed were identified as such and no further key informants were identified, even when asked at the end of each interview. Additionally, this perspective and study are strengthened by the perspective of the employees who participated in focus groups and were asked about organizational and structural factors affecting SPHM participation. 
However, several study strengths also exist. These include the presence of a strong, comprehensive SPHM program at this facility and the willingness of the hospital employees to collaborate with this project (specifically rehabilitation professionals and hospital administration). The presence of an existing but relatively new SPHM program makes it an ideal time to study program effectiveness. This program meets all recommended criteria for an SPHM cited in several resourses. ${ }^{17,19,25,36}$ This includes high levels of equipment purchasing and strong support at the administrative and personnel levels. As previously mentioned, nearly $50 \%$ of the targeted rehabilitation population volunteered to participate in this study and saturation of themes was reached as determined by study investigators.

Additionally, it should be noted that several lessons were learned along the way that were magnified by the timing of data collection during the COVID-19 pandemic. The first three focus groups were held in person several months prior to the existence of COVID-19 (fall 2019). Even with strong recruitment efforts, only the minimum of three participants per focus group was met for each of these three sessions. The last three focus groups were then required to be reorganized for participant safety and were held via Zoom in the spring/summer of 2020. This, in combination with increased incentivization to all participants both past and present, seemed to improve participation. These latter three focus groups had five, eight and three participants respectively. This is most likely due to their already demanding schedules with little time for breaks. A platform such as Zoom that allows participation at a time and place that is convenient for busy healthcare practitioners should be strongly considered for future research.

\section{E. Conclusions and Implications for Future Research}

In conclusion, the results of this single site study help to fill a gap in the literature pertaining to rehabilitation professionals' distinct needs and uses of patient lift equipment, their role within an SPHM program, and the organizational and administrative effects of these programs on this work group. In terms of lift equipment needs and use, the manual standing and raising aid was the favored piece of equipment and several suggestions for improvements to this equipment to make it more useful for rehabilitation purposes were discussed. Additionally, it is important to note that rehabilitation professionals often utilize lift equipment as therapeutic devices and less often as instruments to improve their own workplace safety. Should equipment manufacturers begin to include some of these suggestions for improvement into equipment design, it may increase rehabilitation professionals' use of lift equipment during patient mobility tasks. This would lead to improved practitioner safety among this distinct occupational group.

Rehabilitation professionals overwhelmingly described patient mobility as the primary aspect of their job. Central to these patient mobility tasks was the idea of a therapeutic value to the mobility. However, despite the fact that these patient mobility tasks have been shown to increase the risk of work-related injuries, participants in this study did not perceive work-related pain as injuries and did not report injuries to their employer. Therefore, when measuring the effect of these SPHM programs on this work group, hospital injury records should not be used but rather information gained directly from the workers. Administrators and researchers should be aware that official reporting systems drastically fail to represent the injury experience among rehabilitation professionals. Researchers should also be careful to include the terms work- 
related pain instead of work-related injury when asking about the adverse effects of patient mobility among this work group.

Finally, this study provides a comprehensive assessment of SPHM program perceptions and effects from an organizational perspective that is often lacking in reports on comprehensive workplace program evaluation. These included the various facets of SPHM program administration, including the role of program training and audits in ongoing SPHM success as well as the interface between employees and administration and how this may affect equipment use and injury reporting. Increased communication with workers and incorporation of their needs and ideas into the administrative policies and practices will increase their ability to practically participate in these SPHM programs and, thus, their work-related safety.

Future studies should use our refined recruitment methods for busy hospital employees including virtual interviews and focus groups to overcome scheduling difficulties and consider higher levels of remuneration for participation to compensate for their time lost from work. Additionally, the inclusion of multiple hospital facilities with existing SPHM programs would allow increased depth and breadth of the SPHM needs and participation among this distinct work group. These studies could use the current results to create a mixed-methods approach using both observational or survey data as well as focus groups to explore the various facets of SPHM program success. Studies that actively compare nurses and rehabilitation professionals is also strongly recommended to accurately understand the differences in needs and work habits between these two work groups. 


\section{References}

1. $\quad$ US Department of Labor. BLS Occupation Injury Rate Data 2018.; 2018.

2. (BLS) B of LS. Survey of Occupational Injuries and IIInesses 2018 Chart Package.; 2018.

3. Pompeii LA, Lipscomb HJ, Schoenfisch AL, Dement JM. Musculoskeletal Injuries Resulting From Patient Handling Tasks Among Hospital Workers. Am J Ind Med. 2009;578:571-578.

4. Campo M, Weiser S, Koenig KL, Nordin M. Work-related musculoskeletal disorders in physical therapists: a prospective cohort study with 1-year follow-up. Phys Ther. 2008;88:608-619.

5. Alperovitch-Najenson D, Treger I, Kalichman L. Physical Therapists Versus Nurses in a Rehabilitation Hospital : Comparing Prevalence of Work-Related Musculoskeletal Complaints and Working Conditions. Arch Environ Occup Health. 2014;69:33-39.

6. Darragh AR, Huddleston W, King P. Work-Related Musculoskeletal Injuries and Disorders Among Occupational and Physical Therapists. Am J Occup Ther. 2009;63:351-362.

7. Milhem M, Kalichman L, Ezra D, Alperovitch-Najenson D. Work-Related Musculoskeletal Disorders Among Physical Therapists: a Comprehensive Narrative Review. Int J Occup Med Environ Health. 2016;29:735-747.

8. Darragh, Amy R., Campo M KP. Work-Related Activities Associated with Injury in Occupational and Physical Therapists. Work. 2012;42:1-19.

9. Vieria E, Schneider P, Guidera C, Gadotti IC, Brunt D. Work-related musculoskeletal disorders among physical therapists: a systematic review. J Back Musculoskelet Rehabil. 2016;29:417-428.

10. Serranheira F, Sousa-Uva M, Sousa-Uva A. Hospital nurses tasks and work-related musculoskeletal disorders symptoms: A detailed analysis. Work. 2015;51:401-409.

11. Bork BE, Cook TM, Rosecrance JC, et al. Work-Related Musculoskeletal Disorders Among Physical Therapists. Phys Ther. 1996;76:827-835.

12. US Department of Labor. BLS Occupation Injury Raw Data 2018.; 2018.

13. Veterans Health Administration; Department of Defense. Annex Patient Care Ergonomics Resource Guide : Safe Patient Handling and Movement.; 2001.

14. Department of Veterans Affairs and Veterans Health Administration. VA Directive: Safe Patient Handling Program and Facility Design.; 2010.

15. Department of Health and Human Services and Centers for Disease Control and National Institute for Occupational Safety and Health. Safe Patient Handling Training for Schools of Nursing.; 2010.

16. Department of Health and Human Services and Centers for Disease Control and National Institute for Occupational Safety and Health. Safe Lifting and Movement of Nursing Home Residents.; 2006.

17. Geiger JS. Establishing a physical therapist-driven model of safe patient handling and movement programs in a general hospital. Work. 2013;45:147-160.

18. United States Department of Labor and Occupational Safety and Health Administration. Guidelines for Nursing Homes: Ergonomics for the Prevention of Musculoskeletal Disorders.; 2009.

19. Nelson A, Baptiste A. Evidence-Based Practices for Safe Patient Handling and Movement. Online J Issues Nurs. 2004:2004.

20. Mayeda-Letourneau J. Safe patient handling and movement: A literature review. Rehabil 
Nurs. 2014;39:123-129.

21. Schoenfsch AL, Lipscomb HJ, Pompeii LA, Myers DJ, Dement JM. Musculoskeletal injuries among hospital patient care staff before and after implementation of patient lift and transfer equipment. Scand J Work Environ Heal. 2013;39:27-36.

22. $\mathrm{Li} \mathrm{J}$, Wolf L, Evanoff B. Use of mechanical patient lifts decreased musculoskeletal symptoms and injuries among health care workers. Inj Prev. 2004;10:212-216.

23. Evanoff B, Wolf L, Aton E, Canos J, Collins J. Reduction in Injury Rates in Nursing Personnel Through Introduction of Mechanical Lifts in the Workplace. 2003;457:451-457.

24. Yoder A, Harrison D, Wright K. Outcomes of a Safe Patient Handling Program Implementation. Am J Safe Patient Handl Mov. 2014;4:111-117.

25. Yassi A, Cooper JE, Tate RB, et al. A Randomized Controlled Trial to Prevent Patient Lift and Transfer Injuries of Health Care Workers. Spine (Phila Pa 1976). 2001;26:1739-1746.

26. Harwood KJ, Darragh AR, Campo MA, Rockefeller K, Scalzitti D. A Systematic Review of Safe Patient Handling and Mobility Programs to Prevent Musculoskeletal Injuries in Occupational and Physical Therapists and Assistants. Int J Safe Patient Handl Mobil. 2018;8:46-56.

27. Darragh AR, Campo M, Olson D. Therapy practice within a minimal lift environment: Perceptions of therapy staff. Work. 2009;33:241-253.

28. Darragh AR, Campo MA, Frost L, Miller M, Pentico M, Margulis H. Safe-Patient-Handling Equipment in Therapy Practice : Implications for Rehabilitation. Am J Occup Ther. 2013;67:45-53.

29. Olkowski BF, Stolfi AM. Research Report Safe Patient Handling Perceptions and Practices: A Survey of Acute Care. Phys Ther. 2014;94:682-695.

30. Noble NL, Sweeney NL. Barriers to the Use of Assistive Devices in Patient Handling. Workplace Health Saf. 2017:1-8.

31. Lee SJ, Lee JH. Safe patient handling behaviors and lift use among hospital nurses: A cross-sectional study. Int J Nurs Stud. 2017;74(January):53-60.

32. Waters T, Rockefeller K. Safe Patient Handling For Rehabilitation Professionals. Rehabil Nurs. 2010;35:216-222.

33. Rugs D, Powell-Cope G, Campo M, et al. The use of safe patient handling and mobility equipment in rehabilitation. Work. 2020;66:31-40.

34. Cromie JE, Robertson VJ, Best MO. Work-related musculoskeletal disorders and the culture of physical therapy. Phys Ther. 2002;82(5):459-472.

35. Myers DJ, Schoenfisch AL, Lipscomb HJ. Cultural influences on workplace safety: An example of hospital workers' adoption of patient lifting devices. Saf Sci. 2012;50:494-501.

36. Hignett S. Intervention strategies to reduce musculoskeletal injuries associated with handling patients: a systematic review. Occup Environ Med. 2003;60.

37. Dekker SWA. Patient Safety: A Human Factors Approach. Boca Raton, FL: CRC Press; Taylor and Francis Group; 2011.

38. Schoenfisch AL, Myers DJ, Pompeii LA, Lipscomb HJ. Implementation and adoption of mechanical patient lift equipment in the hospital setting: The importance of organizational and cultural factors. Am J Ind Med. 2011;54:946-954. doi:10.1002/ajim.21001

39. Brewer S, King E, lii BCA, et al. A Systematic Review of Injury / IIIness Prevention and Loss Control Programs ( IPCs ); 2007.

40. van Oostrom S, Driessen M, Franche R, et al. Workplace interventions for preventing work disability. Cochrane Database Syst Rev. 2009;15. 
41. Gensby U, Labriola M, Irvin E, Amick B iii, Lund T. A Classification of Components of Workplace Disability Management Programs: Results from a Systematic Review. J Occup Rehabil. 2014;24:220-241.

42. Tveito T, Sembajwe G, Boden L, et al. Impact of Organizational Policies and Practices on Workplace Injuries in a Hospital Setting. J Occup Environ Med. 2014;56:802-808.

43. Merriam S, Tisdell E. Qualitative Research: A Guide to Design and Implementation. John Wiley \& Sons; 2015.

44. Creswell JW, Poth CN. Qualitative Inquiry and Research Design; 4th Edition. Sage Publishing; 2017.

45. Arjo. https://www.arjo.com/en-us/. Accessed October 13, 2020.

46. Transcribe Audio to Text: Transcription Company \& Website. http://www.rev.com. Accessed October 6, 2020.

47. Gibbs G. Thematic Coding and Categorizing; Analyzing Qualitative Data. London: SAGE Publications, Ltd; 2007.

48. Saldana J. The Coding Manual for Qualitative Researchers. Sage Publishing; 2015.

49. Huhn K, Gilliland SJ, Black LL, Wainwright SF, Christensen N. Clinical Reasoning in Physical Therapy: A Concept Analysis. Phys Ther. 2019;99:440-456.

50. Kucera KL, Schoen AL, Mcilvaine J, et al. International Journal of Nursing Studies Factors associated with lift equipment use during patient lifts and transfers by hospital nurses and nursing care assistants : A prospective observational cohort study. Int $J$ Nurs Stud. 2019;91:35-46.

51. Kanaskie ML, Snyder C. Nurses and nursing assistants decision-making regarding use of safe patient handling and mobility technology: A qualitative study. Appl Nurs Res. 2018;39:141-147.

52. Kitson A, Conroy T, Wengstrom Y, Profetto-McGrath J, Robertson-Malt S. Defining the fundamentals of care. Int J Nurs Pract. 2010;16(4):423-434.

53. Lee S, Faucett J, Gillen M, Landry L. Risk Perception of Musculoskeletal Injury. 2013;62(1):36-44.

54. Holder NL, Clark HA, DiBlasio JM, et al. Cause, Prevalence, and Response to Occupational Musculoskeletal Injuries Reported by Physical Therapists and Physical Therapist Assistants. Phys Ther. 1999;79(7):642-652.

55. Merriam S, Tisdell E. Qualitative Research: A Guide to Design and Implementation. 4th ed. Josey-Bass: A Wiley Brand; 2016.

56. Monaghan HM. Making Safe Patient Handling and Mobility Training Effective. Part 1. What to Teach, Where and When to Teach It and How to Teach It. Int $J$ Safe Patient Handl Mobil. 2019;9(4):143-148.

57. Monaghan HM. Making Safe Patient Handling and Mobility Training Effective. Part 2. Assessing Competent Practice. Int J Safe Patient Handl Mobil. 2020;10(1):37-41.

58. Breslin C, Kyle N, Bigelow P, et al. Effectiveness of health and safety in small enterprises: A systematic review of quantitative evaluations of interventions. J Occup Rehabil. 2010;20(2):163-179.

59. Enos L. Making the Business Case To Initiate, Evaluate, and Sustain Safe Patient Handling Programs: Part 2. Am J Safe Patient Handl Mov. 2011;1(4):22-30.

60. Siddharthan K, Hodgson M, Rosenberg D, Haiduven D, Nelson A. Under-reporting of work-related musculoskeletal disorders in the Veterans Administration. Int J Health Care Qual Assur. 2006;19:461-474. 
61. Lopez SH. Culture Change Management in Long-Term Care : A Shop-Floor View. 2006;34(1):55-80.

62. Gertz C. The Interpretation of Cultures: Selected Essays. New York: Basic Books, Inc; 1973. 


\section{Table: Demographic Information of Focus Group Participants ( $n=25)$}

\begin{tabular}{|c|c|c|c|c|c|}
\hline $\begin{array}{l}\text { Focus } \\
\text { Group }\end{array}$ & $\begin{array}{l}\text { Participant } \\
\#\end{array}$ & Sex & Profession & $\begin{array}{c}\text { Total Work } \\
\text { Experience (Yrs) }\end{array}$ & $\begin{array}{c}\text { Acute Care } \\
\text { Experience } \\
\text { (Yrs) }\end{array}$ \\
\hline 1 & 1 & $F$ & PT & 4 & 4 \\
\hline 1 & 2 & $F$ & PT & 8 & 8 \\
\hline 1 & 3 & $F$ & OT & 9.5 & NR \\
\hline 2 & 4 & $F$ & PT & 8 & 3 \\
\hline 2 & 5 & $F$ & PT & 3 & 3 \\
\hline 2 & 6 & $F$ & PT & 10 & 10 \\
\hline 3 & 7 & $F$ & OT & 12 & 3 \\
\hline 3 & 8 & $F$ & PT & 3 & 2 \\
\hline 3 & 9 & $F$ & PT & 5 & 3.5 \\
\hline 4 & 10 & $F$ & OT & 13 & 13 \\
\hline 4 & 11 & $F$ & OT & 10 & 8 \\
\hline 4 & 12 & $F$ & OT & 8 & 6 \\
\hline 4 & 13 & $F$ & PTA & NR & $7 \mathrm{mo}$ \\
\hline 4 & 14 & $F$ & OT & 26 & 7 \\
\hline 5 & 15 & $F$ & OT & NR & 5 \\
\hline 5 & 16 & $F$ & PTA & NR & 3 \\
\hline 5 & 17 & $F$ & PT & NR & 14 \\
\hline 5 & 18 & $F$ & PTA & NR & 11 \\
\hline 5 & 19 & $F$ & PT & NR & 14.5 \\
\hline 5 & 20 & $F$ & PT & NR & 2 \\
\hline 5 & 21 & $F$ & OT & NR & 3 \\
\hline 5 & 22 & $M$ & PT & NR & 4 \\
\hline 6 & 23 & $F$ & OT & 3 & 2.5 \\
\hline 6 & 24 & $F$ & OT & 10 & 10 \\
\hline 6 & 25 & $\mathrm{~F}$ & OT & 4 & 4 \\
\hline
\end{tabular}

$P T=$ Physical Therapist, $O T=$ Occupational Therapist, $P T A=$ Physical Therapist Assistant $N R=$ Not reported 